\title{
Multiple satellites observation evidence: High-m Poloidal ULF waves with time-varying polarization states
}

\author{
Chao Wei ${ }^{1,2}$, Lei Dai ${ }^{1 *}$, SuPing Duan ${ }^{1}$, Chi Wang ${ }^{1}$, and YuXian Wang ${ }^{1,2}$ \\ 'State Key Laboratory of Space Weather, National Space Science Center, Chinese Academy of Sciences, Beijing 100190, China; \\ ${ }^{2}$ College of Earth and Planetary Sciences, University of Chinese Academy of Sciences, Beijing 100049, China
}

\begin{abstract}
We report multi-spacecraft observations of ULF waves from Van Allen Probes (RBSP), Magnetospheric Multiscale (MMS), Time History of Events and Macroscale Interactions during Substorm (THEMIS), and Geostationary Operational Environmental Satellites (GOES). On August 31, 2015, global-scale poloidal waves were observed in data from RBSP-B, GOES and THEMIS from $L=4$ to $L=8$ over a wide range of magnetic local time (MLT). The polarization states varied towards purely poloidal polarity. In two consecutive orbits over 18 hours, RBSP-A and RBSP-B recorded gradual variation of the polarization states of the poloidal waves; the ratio $\left(\left|B_{a}\right| /\left|B_{r}\right|\right)$ decreased from 0.82 to 0.13 . After the variation of polarization states, the poloidal ULF waves became very purely poloidal waves, localized in both $L$ and MLT. We identify the poloidal wave as second harmonic mode with a large azimuthal wave number $(m)$ of -232 . From RBSP particle measurements we find evidence that the high- $m$ poloidal waves during the polarization variations were powered by inward radial gradients and bump-on-tail ion distributions through the $N=1$ drift-bounce resonance. Most of the time, the dominant free energy source was inward radial gradients, compared with the positive gradient in the energy distribution of the bump-on-tail ion distributions.
\end{abstract}

Keywords: poloidal waves; polarization rotation; bump-on-tail; inward gradient

Citation: Wei, C., Dai, L., Duan, S. P., Wang, C., and Wang, Y. X. (2019). Multiple satellites observation evidence: High- $m$ Poloidal ULF waves with time-varying polarization states. Earth Planet. Phys., 3(3), 190-203. http://doi.org/10.26464/epp2019021

\section{Introduction}

The ultra-low-frequency (ULF) waves are geomagnetic field line oscillations in the frequency range $1 \mathrm{mHz}$ to $1 \mathrm{~Hz}$. Two main categories of ULF waves are poloidal waves and toroidal waves, with wave magnetic field oscillations in the radial and azimuthal directions, respectively.

Poloidal ULF waves can be generated from sources internal or external to the magnetosphere. External sources in the solar wind include the Kelvin-Helmholtz (K-H) instability (Southwood, 1974; Chen L and Hasegawa, 1974; Liu W et al., 2011), dynamic pressure variations (Kessel, 2008; Zong QG et al., 2007, 2009; Zhou XZ et al., 2015), and fluctuations in the foreshock region (Russell et al., 1983; Takahashi et al., 2015). Poloidal ULF waves generated by external sources usually have a low or high azimuthal wave number $m$ and are coupled with strong compressional components (Hughes, 1994; Zong QG et al., 2009, 2017). In general, the wave number $m$ is considered low when $m \sim 1$, intermediate when $m \sim 10$, and large when $m$ is several tens to a few hundreds. Internal sources of poloidal ULF waves include drift-mirror instability (Cheng CZ and Lin CS, 1987), drift resonance and drift-bounce resonance (Southwood, 1976). Poloidal waves generated by internal sources generally have high- $m$ numbers. In high beta plasmas, the

Correspondence to: L. Dai, Idai@spaceweather.ac.cn

Received 13 DEC 2018; Accepted 14 FEB 2019.

Accepted article online 18 MAR 2019.

(C) 2019 by Earth and Planetary Physics. compressional component is coupled to poloidal waves in driftmirror instabilities (Chen $L$ and Hasegawa, 1991). In low beta plasmas, the compressional component vanishes in high- $m$ poloidal waves (Hughes, 1994). RBSP statistical survey shows that compressional Pc 4 poloidal waves are related to solar wind dynamic pressure and that non-compressional Pc 4 poloidal waves are related to decay of the ring current (Dai L et al., 2015).

In the large $m$ limit, the purely poloidal wave is a guided wave (Cummings et al., 1969). Guided poloidal waves are usually generated by drift and drift-bounce resonance instabilities of ring current ions (Southwood, 1976; Engebretson et al., 1992; Oimatsu et al., 2018). Drift resonance and drift-bounce resonance instabilities are responsible for driving odd-number and even-number harmonics of poloidal waves, respectively (Southwood and Kivelson, 1982; Takahashi et al., 2011). Recent RBSP observations provide direct evidence that the fundamental mode poloidal waves are excited by drift resonance with free energy from the gradient in the particle phase space density (Dai L et al., 2013). Such fundamental mode poloidal ULF waves are linked with giant pulsations observed on the ground (Takahashi et al., 2011, 2018a). Previous studies show that second harmonic mode poloidal waves driven by drift-bounce resonance are frequently observed (Hughes et al., 1978; Singer et al., 1982; Takahashi et al., 1990, 2018b). The free energy source for the drift-bounce resonance can be bump-ontail plasma distributions (Hughes et al., 1978; Liu W et al., 2013) and inward radial gradient of the particle phase space density (Min K et al., 2017). 
High- $m$ poloidal waves are frequently observed (e.g, Le et al., 2011; Yeoman et al., 2000). The spatial distribution of high- $m$ poloidal waves is usually localized. Cramm et al. (2000) recorded an event radially localized at about $L=5$ with azimuthal mode number $m=150$. Chi and Le (2015) observed a localized poloidal wave with $m$ number of order 100 and steady wave frequency across local times and $L$ shells. Schäfer et al. (2007) detected radial poloidal ULF oscillations by Cluster with $m \sim 30$. The morphology of the high- $m$ poloidal mode, however, can be complicated. Recently, multiple spacecraft observations have been reported of storm time high-m $(m \sim 100)$ poloidal waves of global scale (Le et al., 2017); these high- $m$ mode waves covered a wide range of $L$ (from 4 to 12) as well as of local times.

Toroidal ULF waves correspond to a response of the magnetosphere to large-scale disturbances (Southwood, 1974; Chen and Hasegawa, 1974). Toroidal waves are usually characterized by multiple harmonic bands in the dynamic frequency spectra (Engebretson et al., 1986). Takahashi et al. (2015) presents a case of a multi-harmonic toroidal wave visible up to the eleventh harmonic. Eigen-frequencies of global-scale toroidal waves have been used to infer plasma mass density of the magnetosphere (Denton and Gallagher, 2000; Menk et al., 1999; Takahashi et al., 2015). In the MHD theory, toroidal waves can be transformed from purely poloidal waves through polarization state variations in nonuniform plasmas (Radoski, 1974). MHD simulations have shown that poloidal waves in the magnetosphere tend to have a finite lifetime and change to toroidal waves (Mann and Wright, 1995). Observational evidence shows that the time for polarization states to vary to toroidal waves is similar to the decay time of the poloidal wave (Sarris et al., 2009). Pc 3 ULF waves due to hot flow anomalies have been observed to exhibit polarization state variations from poloidal mode to toroidal mode (Zhao LL et al., 2017).

Observations of wave polarization may thus yield important information regarding the generation of ULF waves. In this study, we report time-varying polarization states from global poloidal waves to localized and purely poloidal ULF waves. We combine observations from multiple satellites, including Van Allen Probes (RBSP), Magnetospheric Multiscale (MMS), Time History of Events and Macroscale Interactions during Substorm (THEMIS), and Geostationary Operational Environmental Satellites (GOES). The organization of the paper is as follows. In Section 2.1, we show the solar wind and geomagnetic conditions for the event. In Section 2.2, we present observations of the spatial distribution of the waves and the time-varying polarization states from mixed mode to purely poloidal mode. In Section 2.3, we analyze the wave properties during the variation of polarization states. In Section 2.4, we present evidence of the sources of free energy for the second harmonic mode poloidal wave. Section 3 is summary and discussions.

\section{Observations}

\subsection{The Solar Wind and Geomagnetic Activity Context}

Figure 1 presents the solar wind and geomagnetic activity context for our event. The SYM- $H$ index, $A E$ index, and solar wind conditions from August 25 to September 3 are shown. The main phase of a moderate magnetic storm started on August 26, 2015, as indicated in Figure $1 \mathrm{a}$. The SYM- $H$ decreased to the minimum value $-90 \mathrm{nT}$ late on August 27. The storm's main phase lasted for about 2 days, from August 26 to August 28. The recovery phase developed for about 4 days, from August 28 to September 01. During the storm's main phase and its early recovery phase, strong and continuous $A E$ activities are seen (Figure $1 \mathrm{~b}$ ). Figures $1 \mathrm{c}-$ 1e present solar wind proton number density $\left(N_{\mathrm{p}}\right)$, velocity $\left(V_{\mathrm{p}}\right)$, and dynamic pressure $\left(P_{\text {dyn }}\right)$. Strong variations of $P_{\text {dyn }}$ are seen in the main phase. In the recovery phase, both $A E$ and $P_{\text {dyn }}$ are small. The observed ULF waves occur in the late recovery phase and quiet time. The two vertical dashed lines indicate the interval when ULF waves are observed with time-varying polarization states from 23:00 UT on August 31 to 17:00 UT on September 1. The time-varying polarization states lasted for two RBSP orbit (18 hours) in a quiet time, during which no significant variations are seen in the solar wind or geomagnetic activities.

\subsection{Spatial Distribution of ULF Waves and Polarization Variation}

Figure 2 is an overview of the spatial distribution of observed ULF waves. Figure 2a shows the observation of initially poloidal ULF waves, by RBSP-B, GOES-13 and THEMIS-A, in the $x y$-plane of GSM coordinate near the day 2015-08-31. Locations of observed ULF wave activity are marked with colors. The red corresponds to the observation of waves by RBSP-B from 23:00 UT on August 31 to 02:30 UT on September 1. The pink corresponds to the ULF waves observed by GOES-13 from 23:20 UT on August 31 to 02:00 UT on September 1 . The green corresponds to the waves obseved from THEMIS-A from 23:30 UT to 02:00 UT. The corresponding distributions of the ULF waves in $L$ shell $(L)$ and magnetic local time (MLT) are shown in Figure $2 b$ and $2 c$. We use a dipole magnetic field model in our study. $L$ in this paper refers to " $L$ shell", which is the Mcllwain $L$. The ULF waves are observed in the inner magnetosphere at $L<5$; we believe that Mcllwain $L$ and Roederer $L$ are similar in this range. The time span of Figure $2 b$ and $2 c$ is from 20:00 UT on August 31 to 04:00 UT on September 01. The colors corresponding to each spacecraft are consistent with those in Figure 2a. From Figure $2 \mathrm{~b}$ we can see that the initially observed poloidal waves covered a wide range of $L$, from 4 to 8 . THEMIS, RBSP, and GOES observations are well separated in MLT, ranging from dawn to afternoon to dusk-night sectors. Figure $2 \mathrm{c}$ shows that the waves were located over a wide range of MLT, from 7 to 20 .

The schematics of RBSP and MMS during the polarization state variations of the ULF waves in the GSM $x y$-plane are shown in Figure $2 \mathrm{~d}$. The orbit period of RBSP spacecraft is about 9 hours. RBSP-A observed the ULF waves on two consecutive orbits about 9 hours apart: at 06:00-09:35 UT and at 14:50-16:00 UT. RBSP-B observed the ULF waves at 08:00-11:00 UT and 16:50-17:10 UT, also on two consecutive orbits. The orbits of RBSP-A and RBSP-B are very close to each other. MMS observed the ULF waves in a similar location, at 06:20-08:00 UT. The MMS orbit was close to that of RBSP-A during this interval. The spatial distribution of the waves in $L$ and MLT during the polarization state variations is presented in Figures 2e and 2f. The colors corresponding to each spacecraft are consistent with those in Figure $2 \mathrm{~d}$. As indicated in Figure 2e, the $L$ shell coverage of the ULF waves from 06:00 to 

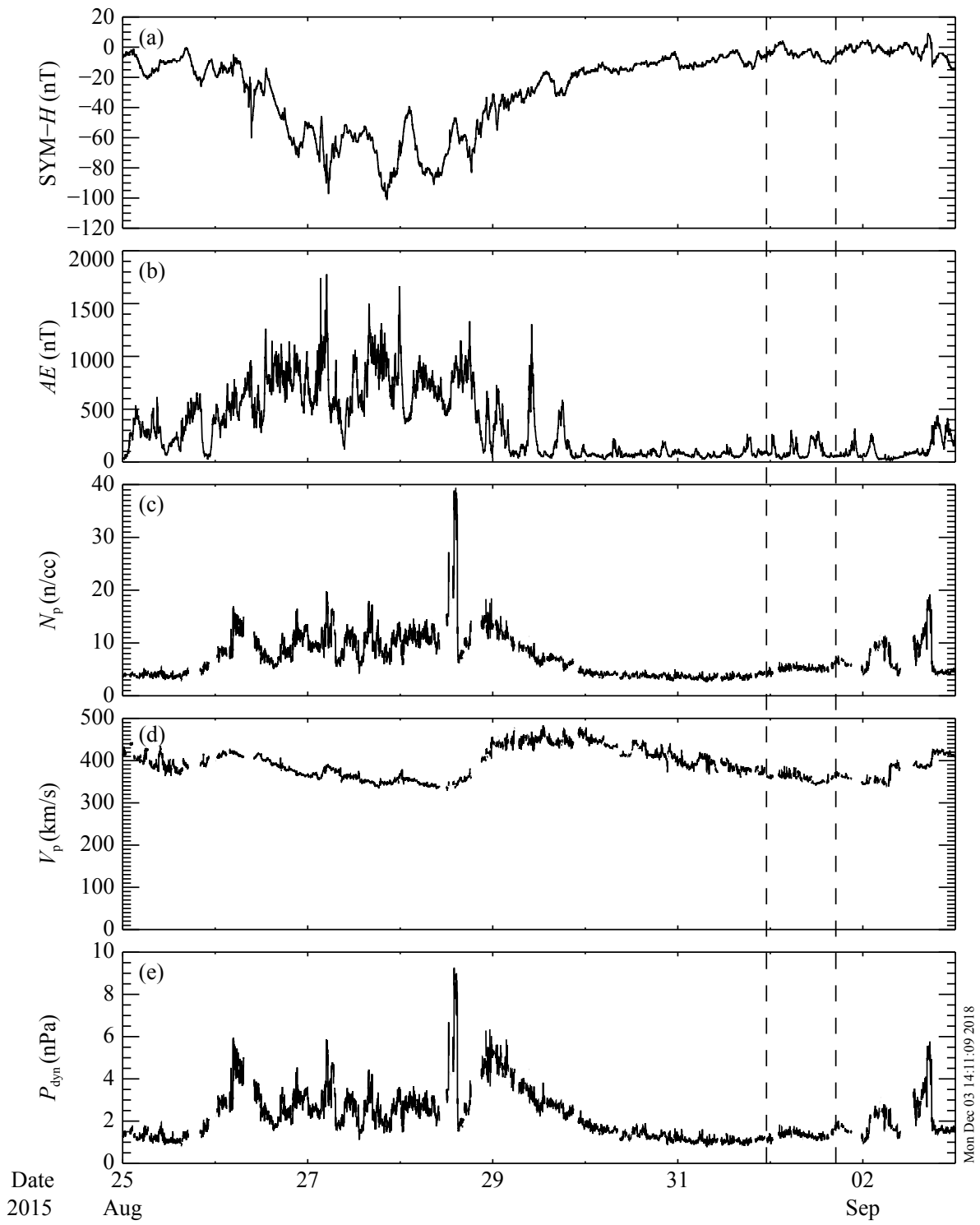

Figure 1. From the top: (a) Dst index; (b) $A E$ index; (c) proton number density in solar wind; (d) solar wind speed; (e) solar wind dynamic pressure. Vertical dash lines indicate the intervals of wave observations.

11:00 UT during the first orbit of RBSP is from 4.0 to 7.6. During the second RBSP orbit, the range of $L$ shell coverage became much smaller (4.3-5.5). From Figure 2f, the MLTs of the wave during the first and the second RBSP orbits are $\sim 13.4-16.5$ and 13.4-14.6, respectively. The $L$ and MLT extent of the waves are much smaller than those of the earlier global-scale waves. The ULF waves become more localized in both $L$ and MLT during the polarization state variations. The localization of the ULF waves may significantly affect the resonance interaction with particles ( $\mathrm{Li}$ L et al., 2017).

We examine wave power spectra and waveforms during the periods of polarization state variation. In Figure 3 we present the Fourier dynamic spectra from 20:00 UT on August 31 to 24:00 UT on September 01 of the wave magnetic field from Electric and Magnetic Field Instrument Suite and Integrated Science (EMFISIS) (Kletzing et al., 2013) of RBSP-A and RBSP-B. The 3-D DC electric field data from EFW (Wygant et al., 2013) of RBSP need to be ob- tained from the $E \cdot B=0$ assumption and are unavailable for this event. The magnetic field is transformed into a magnetic-fieldaligned coordinate system, with $a$ being azimuthally eastward, $p$ being along the 1000s average magnetic field line, and $r$ being radially outward. The dynamic power spectra of the wave fields $B_{r}$, $B_{a}$ and $B_{p}$ are analyzed, with a running FFT time window of $20 \mathrm{~min}$. The lag between running FFT time windows is $1 \mathrm{~min}$. ULF wave activities in the Pc4-PC5 range were detected five times in similar locations by the two RBSP. RBSP-B observed the initial waves for the first time from 23:00-24:00 UT on August 31; the poloidal component was a bit stronger than the toroidal component. ULF waves were again observed by RBSP-A at 06:00-09:35 UT. In the third observation (by RBSP-B at 08:00-11:00 UT), the toroidal component appeared significantly weaker than the poloidal component. During the last two observations (by RBSP-A at 14:50-16:00 UT and by RBSP-B at 16:50-17:00 UT), the wave polarization had become nearly purely poloidal. 
(a)

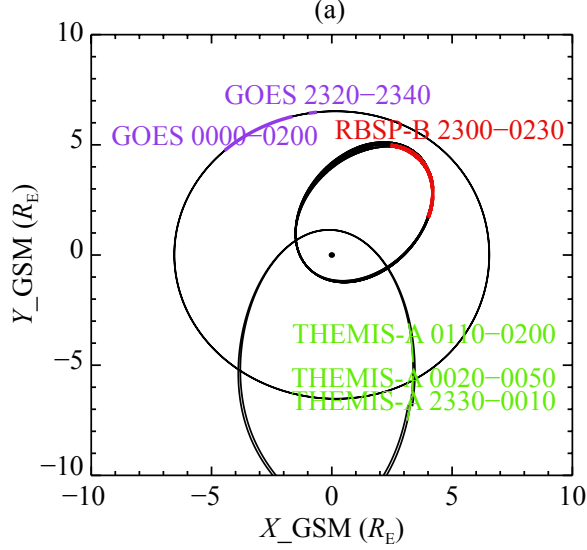

(b)

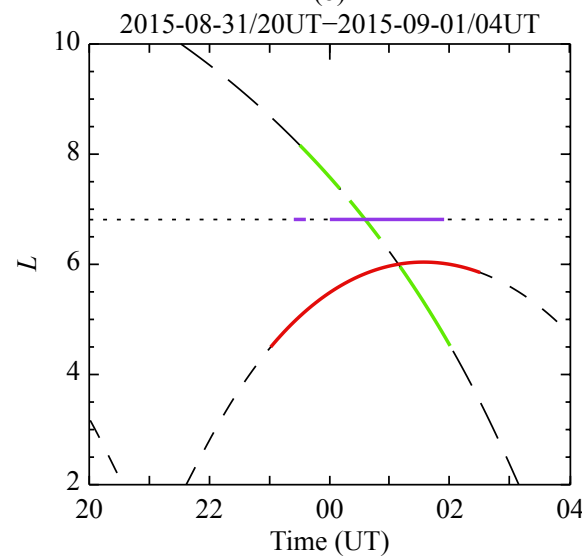

(c)

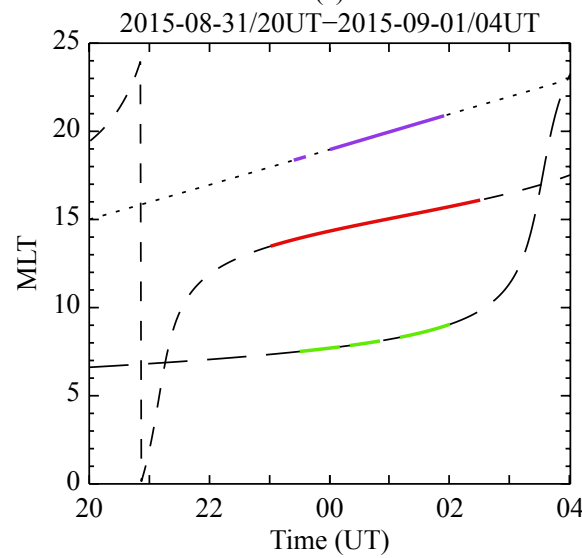

(d)

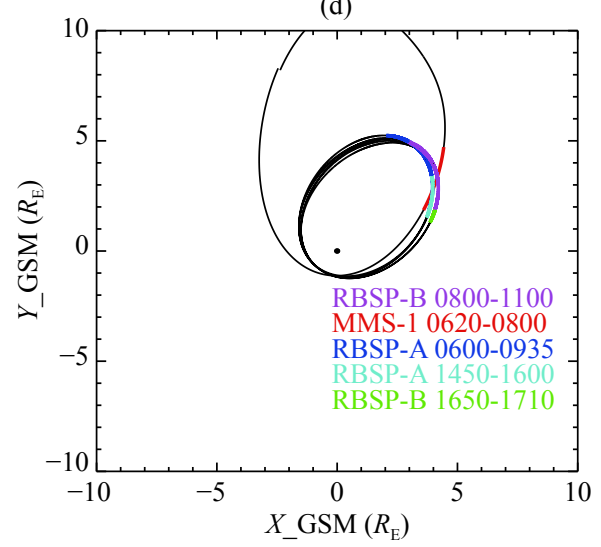

(e)

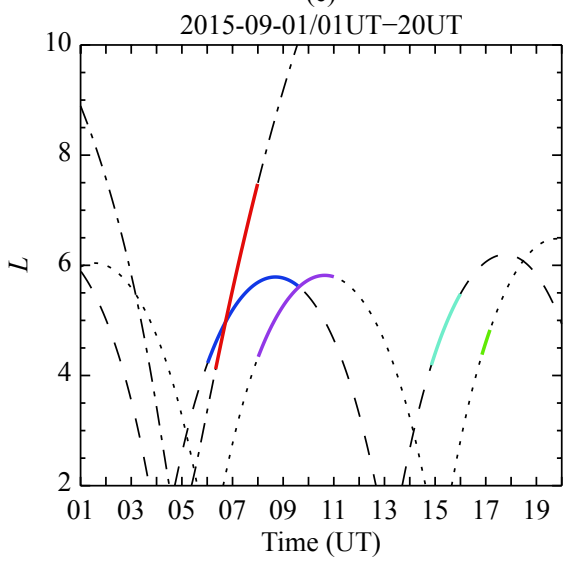

(f)

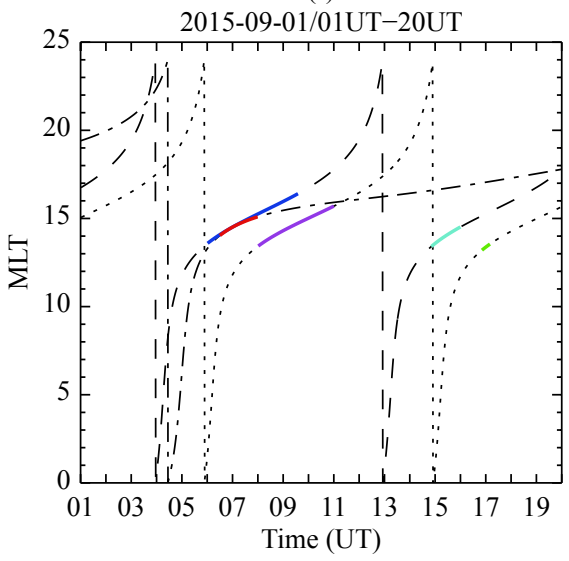

Figure 2. Schematics of location of the observed ULF waves in L and MLT. (a)-(c) Observations of ULF waves from 23:00 UT August 31 to 02:30 UT September 01. Red line indicates the orbit of RBSP-B; Pink line indicates the orbit of GOES-13; Green line indicates the orbit of THEMIS-A. (d)-(e) Observations of ULF waves from 01:00 to 19:00 UT September 01. Dark blue and light blue indicate the two orbits of RBSP-A; Pink and Green indicate the two orbits of RBSP-B; the Red line indicates the orbit of MMS-1.

The observations summarized in Figures 2 and 3 demonstrate that the initially poloidal mode is of global scale and was probably generated by fluctuations in the solar wind upstream. The poloidal wave is generally coupled with some toroidal component under the condition of finite $m$ number.

Figure 4 presents the time-varying polarization states in hodogram. Five 10-min intervals of $B_{a}$ and $B_{r}$ in the $0.005-0.015 \mathrm{~Hz}$ range are analyzed. The spacecraft locations during these intervals were similar. The $L$ shell range was 4.5-5.8; MLT varied from
13.3 to 15 . The interval in Figure $4 a$ is the first observation of the initially poloidal waves from RBSP-B (00:10-00:20 UT on September 01). In this interval, the toroidal polarity was strong; $\left|B_{a}\right| /\left|B_{r}\right|$ $\sim 0.82$. Figure $4 \mathrm{~b}$ is the hodogram of the interval 06:35-06:45 UT from RBSP-A. During this interval, the toroidal mode appears weaker; $\left|B_{a}\right| /\left|B_{r}\right| \sim 0.54$. Two hours later, RBSP-B observed a smaller polarization $\left(\left|B_{a}\right| /\left|B_{r}\right| \sim 0.39\right)$ of the waves at about the same location. In the last two RBSP orbits in Figure $4 d$ and 4 e, RBSP-A observed a polarity with more poloidal components $\left(\left|B_{a}\right| /\left|B_{r}\right| \sim 0.32\right)$. 


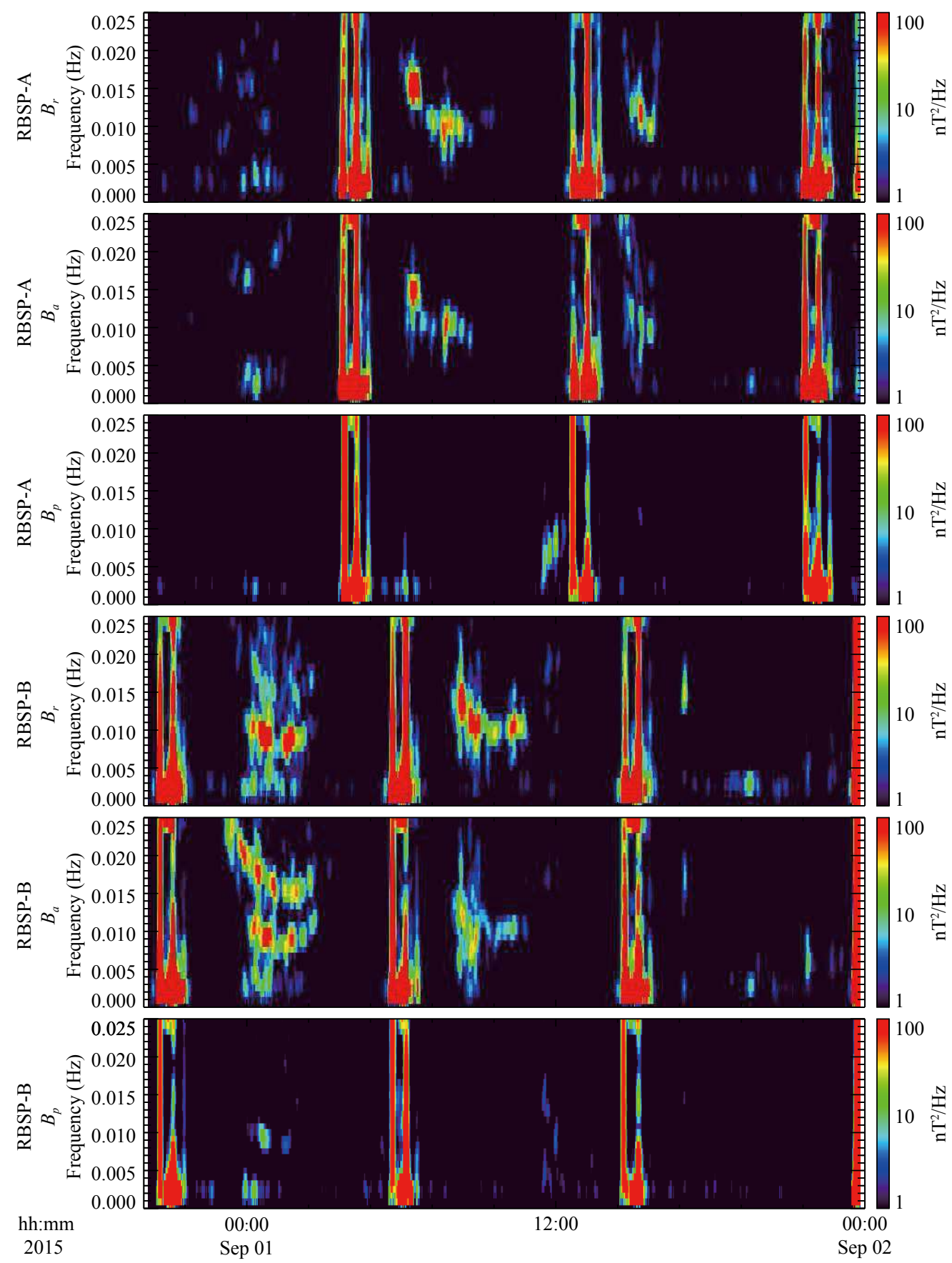

Figure 3. Observations of the dynamic power spectra of $B_{r}, B_{a}$, and $B_{p}$ by RBSP-A and RBSP-B from 23:00 UT August 31 to 24:00 UT September 1.

Following RBSP-A 1.5 hours later, RBSP-B observed a polarity of $\left|B_{a}\right| /\left|B_{r}\right| \sim 0.13$. The hodogram results are consistent with the features described in Figures 2 and 3. A poloidal wave is first observed to be coupled with some toroidal component. Then, as recorded over 18 hours by two RBSP spacecrafts in two consecutive orbits, the ULF waves gradually become purely poloidal.

\subsection{Second Harmonic Poloidal Wave With High-m Number} During the variations of polarization state, MMS encounters the poloidal waves on 06:20-08:00 UT September 1 in the out-bounce at $L=4-6$. We analyze the wave properties from the electric field (Lindqvist et al., 2016) and magnetic field measurements (Russell et al., 2016; Torbert et al., 2016) observed on MMS-1. Figure 5 presents the filtered waveform of $E$ and $B$ in the $0.005-0.025 \mathrm{~Hz}$ band from MMS-1 on 06:30-07:00UT. $B_{r}, B_{a}, B_{p}$ are plotted in the first panel; $E_{r}$ and $E_{a}$ are plotted in the second panel. $B_{r}$ and $E_{r}$ are shifted upward $5 \mathrm{nT}$ and $5 \mathrm{mV} / \mathrm{m}, B_{p}$ is shifted downward $5 \mathrm{nT}$.

The harmonic mode of poloidal oscillations can be identified from the phase difference between $B_{r}$ and $E_{a}$. For even (odd) harmonics, the phase of $E_{a}$ leads (lags) $B_{r}$ by 90 degree for a spacecraft located slightly north of the magnetic equator (Singer et al., 1982; Takahashi et al., 2011). The $B_{r}-E_{a}$ phase relation is opposite in the southern magnetosphere. It is seen from Figure 5 that the phase of $E_{a}$ lags $B_{r}$ by 90 degrees in this event. Combined with the fact 
(a)

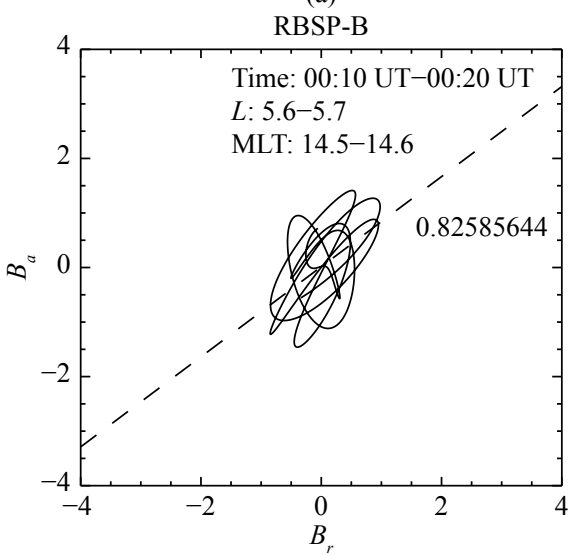

(c)

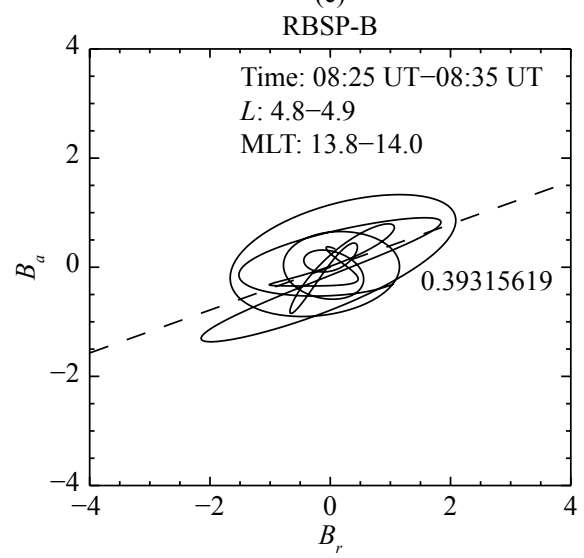

(b)

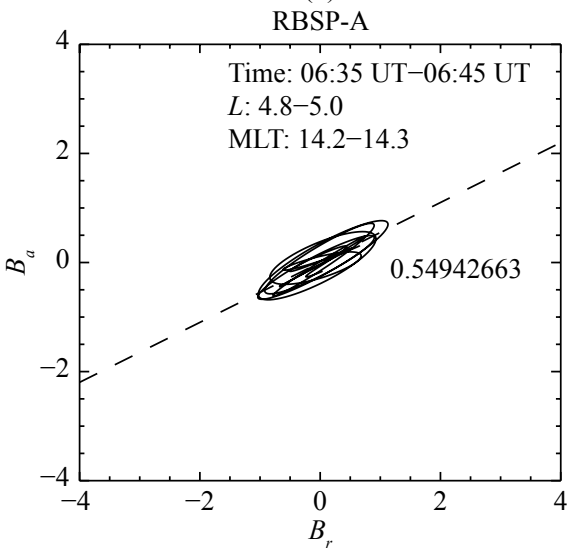

(d)

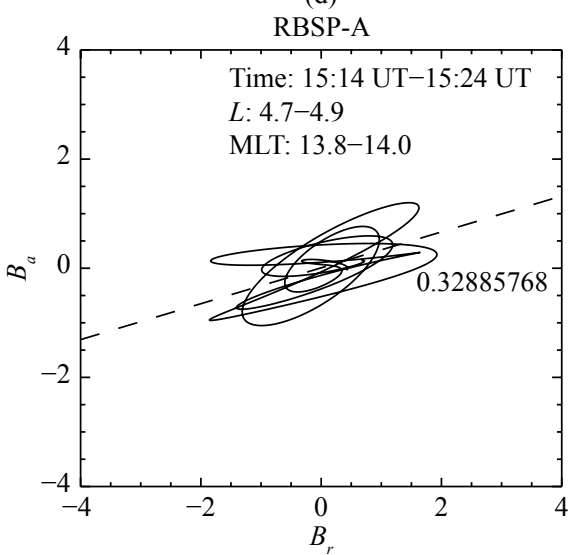

(e)

RBSP-B

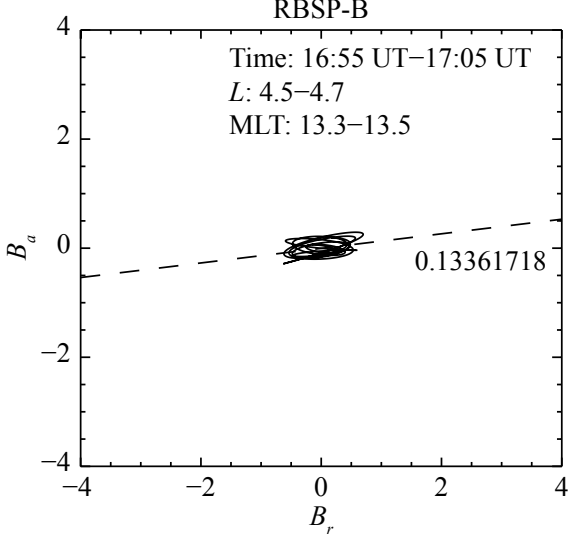

Figure 4. (a)-(e) The hodogram analysis of $B_{r}$ and $B_{a}$ from RBSP-A and RBSP-B during the polarization state variation. Dashed lines are the least squares fits.

that MMS is south of the magnetic equator, the observed wave is an even-number harmonic mode. Considering the value of the eigen-frequency, we conclude that the observed wave is a poloidal standing wave of second harmonic mode.

We use timing from multiple spacecraft measurements of MMS to infer the azimuthal wave number $m$. Figure 6 a show the filtered time series $(0.005-0.025 \mathrm{~Hz})$ of $B_{r}$ and $E_{a}$ of MMS-1, MMS-2, and MMS-3 from 06:35 to 06:50 UT. The peak value of the dynamic power spectra is around $0.013 \mathrm{~Hz}$. The four MMS spacecrafts are separated by less than a few $100 \mathrm{~km}$. During this interval MMS-1,
MMS-2, and MMS-3 were close to each other in $L$. The orbit of MMS-4 was more separated $(\sim 200 \mathrm{~km})$ from the orbit of other three MMS. Poloidal ULF waves may have spatial structures in terms of the Alfven resonator that affect the wave profile in the radial direction (Mager and Klimushkin, 2013; Klimushkin, 1998; Klimushkin et al., 2004; Leonovich and Mazur, 1995). The resonator may cause differences in amplitude and phase across different $L$. Such differences due to radial separation can affect the timing result of the azimuthal wave number $m$. To achieve a more reliable result for the azimuthal wave number $m$, we select data from MMS-1, MMS-2, and MMS-3 for the analysis. From Figure $6 a, a$ 


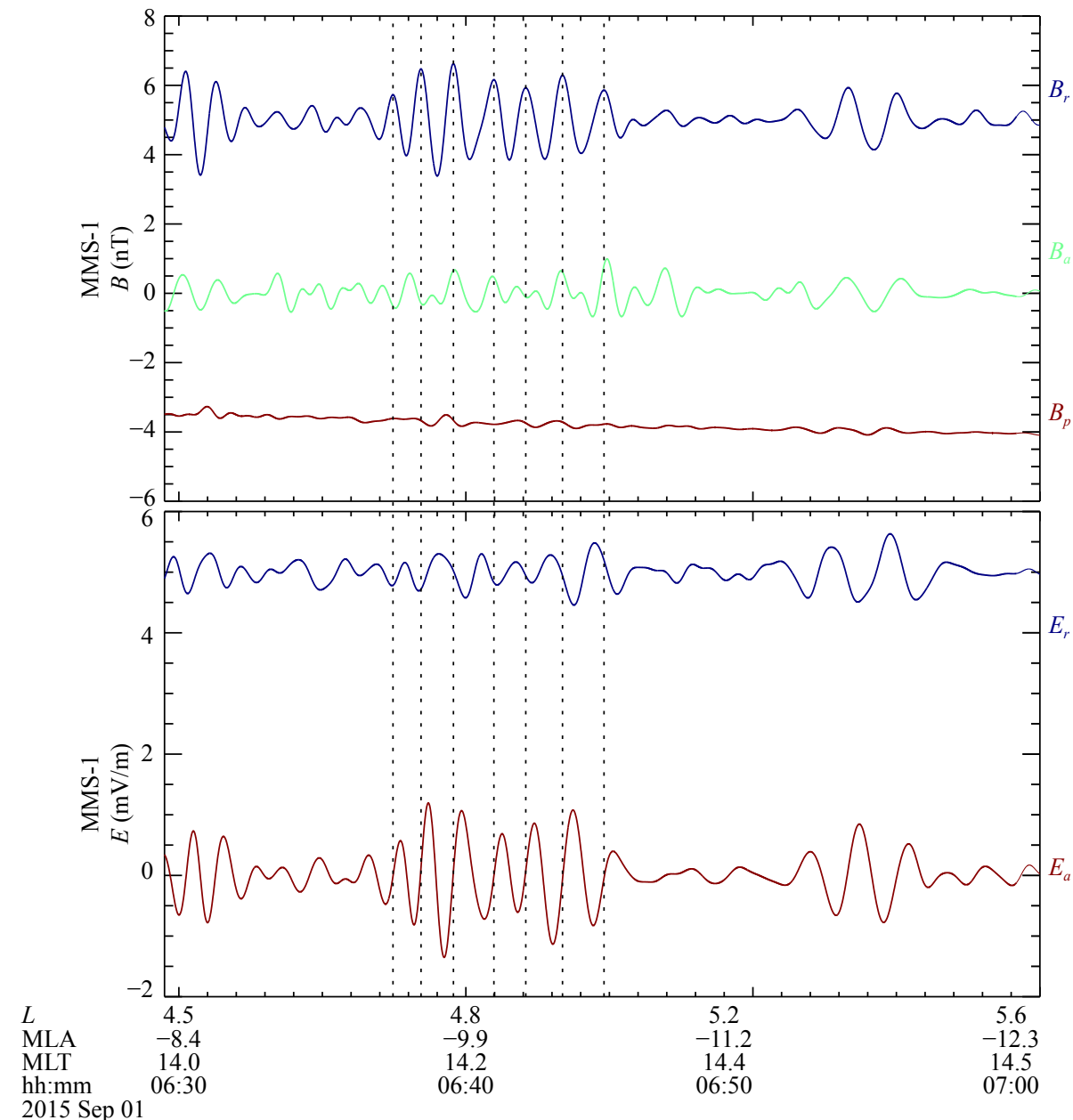

Figure 5. MMS1 observations of the ULF poloidal waves. Waveforms of $B_{r}, B_{a}, B_{p^{\prime}} E_{r}$ and $E_{a}$ are shown.

clear time lag of $B_{r}\left(E_{a}\right)$ can be visually seen between the waveforms from MMS-1, MMS-2, and MMS-3. From the examination of the waveforms, we can estimate qualitatively that the wavelength is of the order of the spacecraft separation $(\sim 100 \mathrm{~km})$. The timing results from $B_{r}$ and $E_{a}$ are nearly the same. Figure $6 \mathrm{~b}$ shows the time lags versus the azimuthal separation distances of the spacecraft. The timing between MMS-1-MMS-2, MMS-1-MMS-3, MMS2-MMS-3 are 10.2 s, $90.4 \mathrm{~s}$ and 100.1 s, respectively; the azimuthal separation between MMS-1-MMS-2, MMS-1-MMS-3, MMS2-MMS-3 are $93.0 \mathrm{~km}, 162.2 \mathrm{~km}, 255.4 \mathrm{~km}$, respectively. The azimuthal wavelength from timing is $172 \mathrm{~km}$, corresponding to $m$ $=-232$. We analyze the error estimation of the azimuthal wave number $m$. The error in the fitted slope of the line is 0.143 . The error of $m$ is calculated to be 75 considering that $m$ is inversely proportional to the slope. The second harmonic poloidal wave is of very high $m$ number $(m=-232 \pm 75)$ and its propagation is westward.

\subsection{Free Energy Source for the High- $m$ Poloidal Waves}

The second harmonic poloidal waves lasted for more than 18 hours with time-varying polarization states. We further investigate the free energy for the waves. The analysis for RBSP-A is presented in Figure 7. We use measurement of ion omnidirectional flux spectra from the ECT instrument (Funsten et al., 2013) to calculate the phase space density $f$ for ions with energy $W$ from 1 $\mathrm{keV}$ to $50 \mathrm{keV}$. There is a close relationship between the differential particle flux $K$ and the particle phase space distribution $f$,

$$
K=\frac{v^{2}}{m_{\mathrm{p}}} \cdot f,
$$

where $K$ is the differential particle flux, $m_{\mathrm{p}}$ is mass of the measured particle, and $v$ is the particle velocity. Two examples of ion phase space density at 07:50:41 and 15:17:03 UT, respectively, from the two consecutive orbits of RBSP-A are shown in Figures $7 a$ and $7 \mathrm{~b}$, in which the bump-on-tail ion distribution function is identified as a positive $\mathrm{d} f / \mathrm{d} W$ gradient. The maximum $W_{\max }$ and minimum $W_{\min }$ energies of the positive gradient region are indicated by dashed lines. The energy for the bump is obtained as $W_{\text {bump }}=\left(W_{\max }+W_{\min }\right) / 2$. The bump energy $W_{\text {bump }}$ is $7057 \mathrm{eV}$ in Figure $7 \mathrm{a}$ and $9158 \mathrm{eV}$ in Figure $7 \mathrm{~b}$.

The bump-on-tail distribution in ring current ions is an important free energy source for the second harmonic poloidal wave (Hughes et al., 1978; Liu W et al., 2013; Takahashi et al., 2018b). And bump-on-tail instability is a very common instability in plasma physics (Fitzenreiter et al., 1984; Berk et al., 1995). Bumpon-tail ion spectra in our study were first reported in Hughes et al. (1978). The bump-on-tail ion spectra correspond to a positive slope $\left(\mathrm{d} f /\left.\mathrm{d} W\right|_{\mu, L}>0\right)$ in the plasma velocity distribution. ULF 

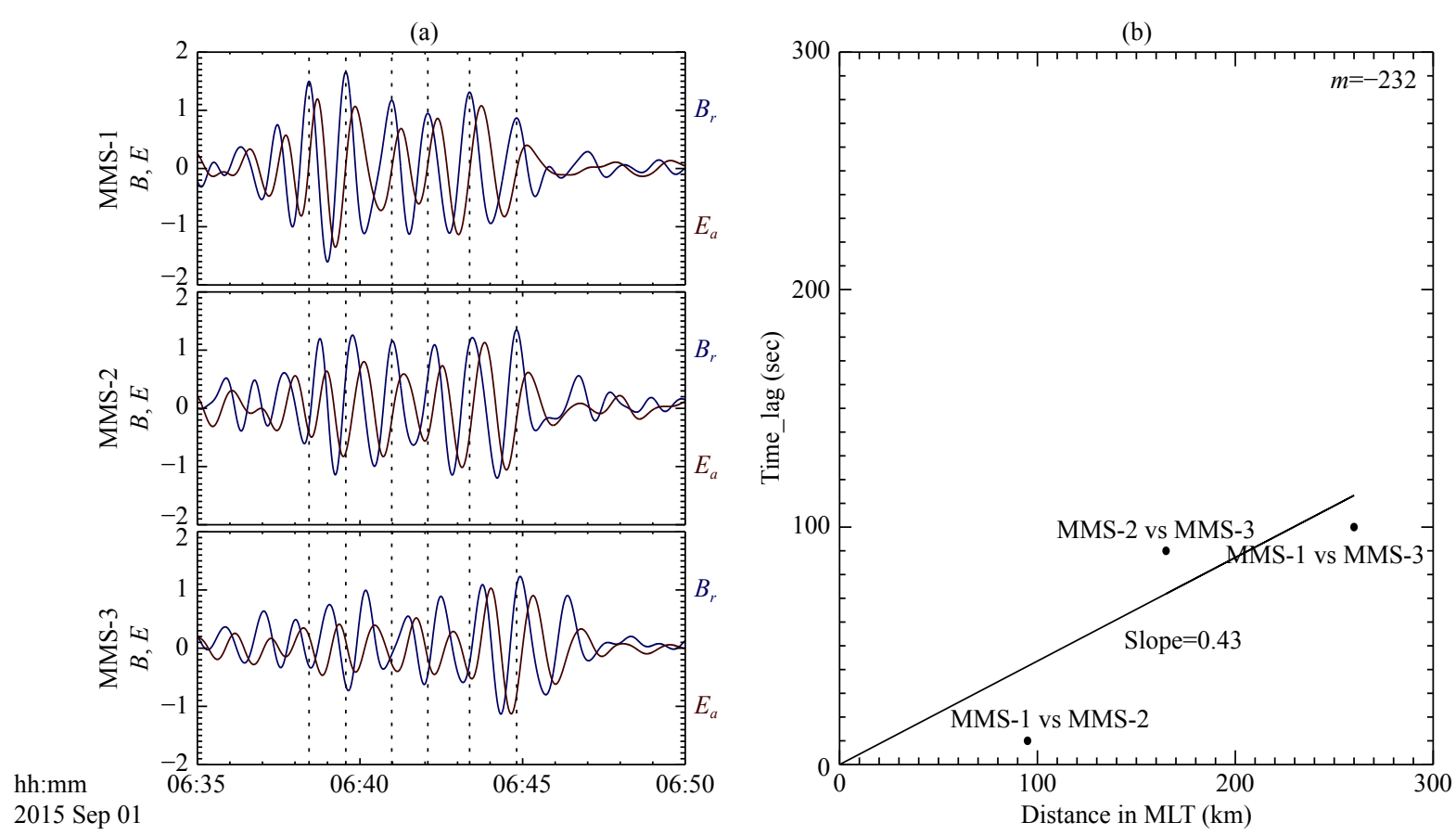

Figure 6. (a) Filtered (0.005- $0.025 \mathrm{~Hz}$ ) waveforms of $B_{r}$ (in blue) and $E_{a}$ (in red) from MMS-1, MMS-2, and MMS-3. (b) Observed time lags in $B_{r}$ versus longitudinal separations between MMS satellites.

waves may be excited by a bump-on-tail velocity distribution. 10 $\mathrm{keV}$ ions are probably injected into the storm and substorm. The process of particle injection can be from different regions with different energies and different PSD. The large-scale injection may be adiabatic and the small-scale injection may be non-adiabatic. This complexity may lead to a reverse of the plasma energy distribution's slope. In our event, the bump energy of the ions is around 6-12 keV, as shown in Figure $7 \mathrm{c}$ and $7 \mathrm{~d}$. The phase space density spectrum of protons shows a persistent existence of 6$12 \mathrm{keV}$ ions associated with the waves. The bump-on-tail ions can drive second harmonic poloidal waves through the drift-bounce resonance.

The condition of drift-bounce resonance is $\omega-m \omega_{D}=N \omega_{B}$, where $\omega$ and $m$ are the wave frequency and azimuthal wave number, respectively, and $\omega_{D}$ and $\omega_{B}$ are the frequencies of particle drift and bounce motion, respectively, and $\mathrm{N}$ is the resonance number. $\omega_{B}$ is described as (Roederer and Hones, 1970)

$$
\omega_{B} \approx \frac{\sqrt{\frac{2 W}{m_{\mathrm{i}}}}}{4 L R_{\mathrm{E}}(1.3-0.56 \sin \alpha)},
$$

in a dipole field, where $W$ is particle energy, $m_{\mathrm{i}}$ is the ion mass, and $a$ is the particle's equatorial pitch angle. $N$ is an integer. $N=0$ is the drift-resonance and $N= \pm 1$ is the drift-bounce resonance. As Southwood (1976) points out, the $N=-1$ particles are more energetic than the $N=1$ particles. The approximate solutions for the drift-bounce are $\omega \simeq \omega_{B}(N=+1)$ and $m \omega_{D} \simeq \omega_{B}(N=-1)$. In our event, the free energy is provided by the low energy $(\sim 10 \mathrm{keV})$ bounce resonance of $N=+1$. The resonance condition is $\omega \simeq \omega_{B}$. This scenario is consistent with the westward propagation of the poloidal wave as obtained in the previous section.
More evidence for the $N=1$ bounce resonance is provided in the last panel of Figures $7 c$ and $7 d$. We compare the observed eigenmode frequency, the eigen-frequency from the resonance condition $\omega \simeq \omega_{B}$, and the modeling eigen-frequency expected from the second harmonic poloidal waves. The modeling eigen-frequency for the second harmonic mode is obtained as follows. Cummings et al. (1969) and Orr and Matthew (1971) have shown that the harmonic eigen-frequencies in a dipole magnetic field can be solved numerically. Cummings et al. (1969) uses an idealized model with a background magnetic field of dipole and the plasma density of power law to calculate the eigen-frequency at $6.6 R_{\mathrm{E}}$. Orr and Matthew (1971) study the eigen-frequencies for different geocentric distances and provide the relationship between eigen-frequency and $L$ value. Following the approach of Orr and Matthew (1971) and Liu W et al. (2013), we use the modeling relation

$$
f_{\mathrm{q}} \propto \frac{1}{\left(\sqrt{n_{\mathrm{eq}}} L^{4}\right)}
$$

for second harmonic poloidal waves, where $f_{\mathrm{q}}$ is local eigen-frequency, $n_{\mathrm{eq}}$ is the proton density at the magnetic equator, and $L$ is the $L$-shell value. Using this relation, we plot the modeling eigenfrequency based on the observed plasma density. As shown in the last panel of Figures $7 c$ and $7 d$, the observed eigenmode frequency (black) matches well with the eigen-frequency of the resonance condition (red) and the modeling eigen-frequency (green) in the presence of the waves. We analyze the average relative errors between observation eigen-frequency, modeling eigen-frequency, and resonance eigen-frequency. The errors between the frequencies for the whole event interval in Figure $7 \mathrm{c}$ are $21 \%, 8 \%$, $14 \%$; in Figure $7 d$ they are $35 \%, 25 \%, 10 \%$. We think average relative error under $35 \%$ constitutes good agreement and supports the scenario that the $\sim 10 \mathrm{keV}$ ions may provide energy to the ei- 
(a)

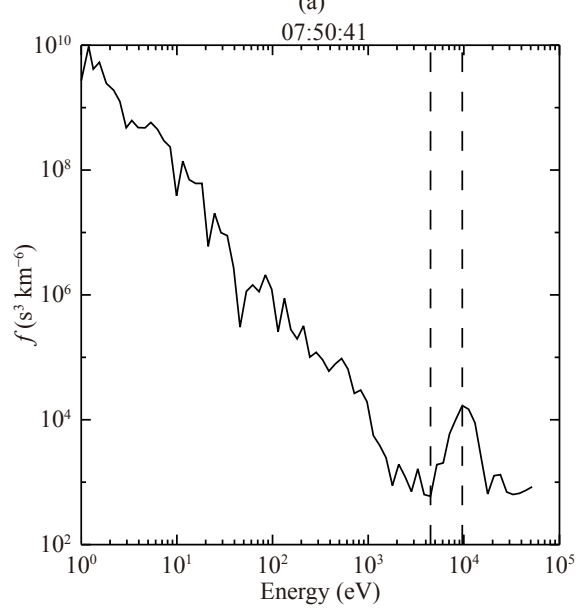

(c)

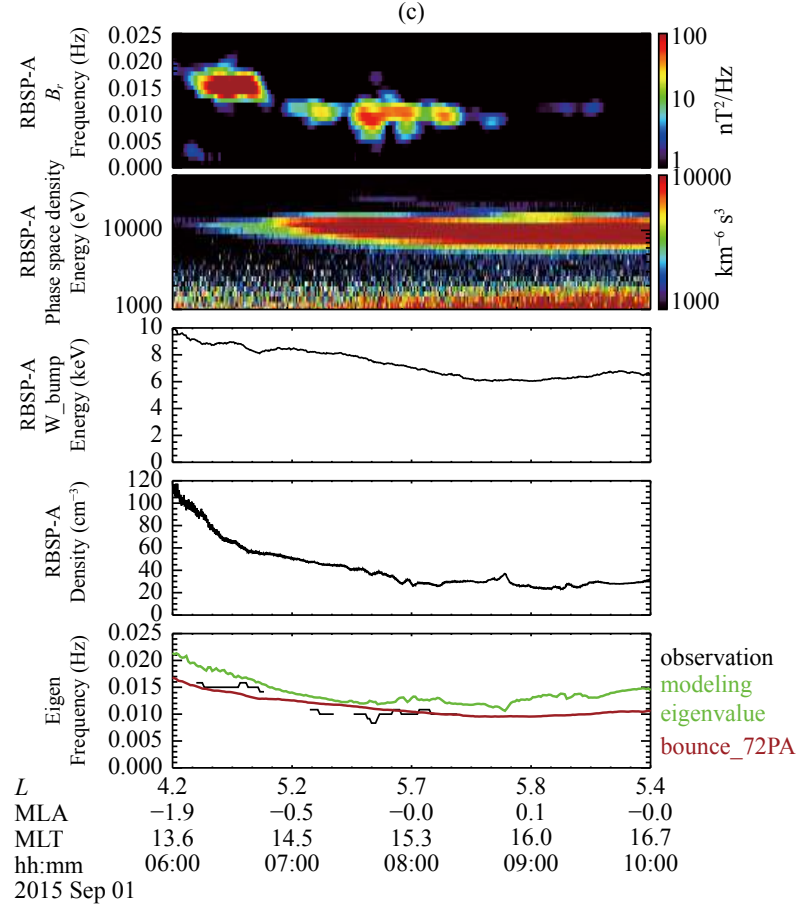

(b)

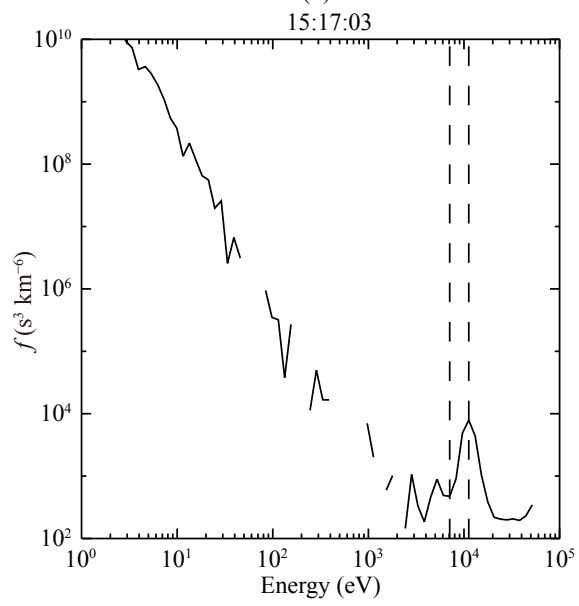

(d)

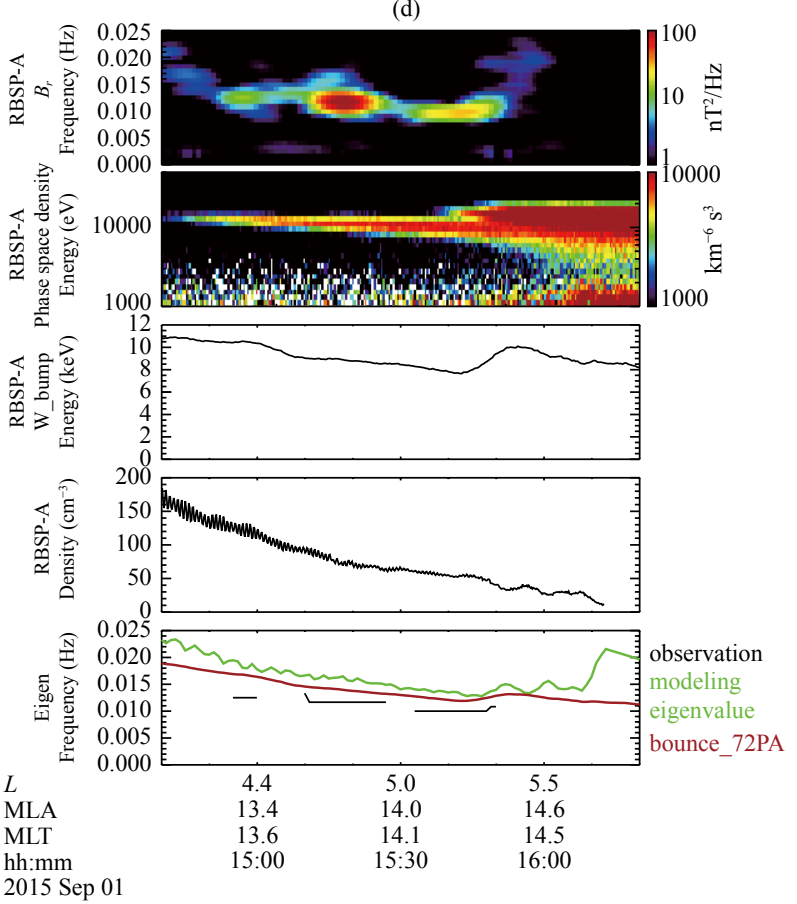

Figure 7. (a)-(b) Proton phase space density measured at 07:50:41 and 15:17:03 UT by RBSPA. (c)-(d) Observations of the waves in the first orbit and the second orbit of RBSP-A. Panels from the top are wave power spectra of $B_{r}$, proton phase space density, energy of the bump distribution, electron density inferred from RBSP spacecraft potential, and eigenmode frequencies from observed wave (black), from modeling of the second harmonic poloidal wave (green), and from the bounce frequency (red) of protons at the energy of the bump.

genmode through the $N=1$ bounce resonance. Some difference between modeling and observation may come from uncertainty in the modeling eigenfrequency. The modeling eigenfrequency assumes some specific form of global density distribution. Our observations include measurement of the density only near the equatorial plane, which is not exactly the same as the modeling density. After 09:00-10:00 UT in the first orbit and 16:00 UT in the second orbit, the modeling eigen-frequency of local field lines gradually deviates from the bounce frequency of $10 \mathrm{keV}$ ions. Under this condition, energy can not be transferred from particles to waves. This probably leads to the decrease of the wave activity in these intervals.

Figure 8 analyzes, in format similar to that of Figure 7, the bumpon-tail distributions observed by RBSP-B. The dynamic power spectra of $B_{r}$, ion phase space density, bump energy, electron density, and comparison of eigen-frequencies are shown. RBSP-B detects wave activities three times. The $\sim 10 \mathrm{keV}$ bump-on-tail ions are present in all intervals. The errors between the frequencies for the whole event interval in Figure $8 \mathrm{a}$ are $13 \%, 25 \%, 14 \%$, in Figure $8 \mathrm{~b}$ are $20 \%, 25 \%, 8 \%$, in Figure $8 \mathrm{c}$ are $4 \%, 31 \%, 30 \%$. Similar to the results of RBSP-A, the observed eigenmode frequency (black) agrees well with the eigen-frequency of the resonance condition (red) and the modeling eigen-frequency (green). In the last orbit on 16:50-17:10 UT of September 1, the decreasing intensity of bump-on-tail ions corresponds to a decrease of wave intensity. Evidence from both RBSP spacecraft supports that $\sim 10 \mathrm{keV}$ ions through the $N=1$ drift-bounce resonance contribute to the generation of the poloidal waves. 
(a)

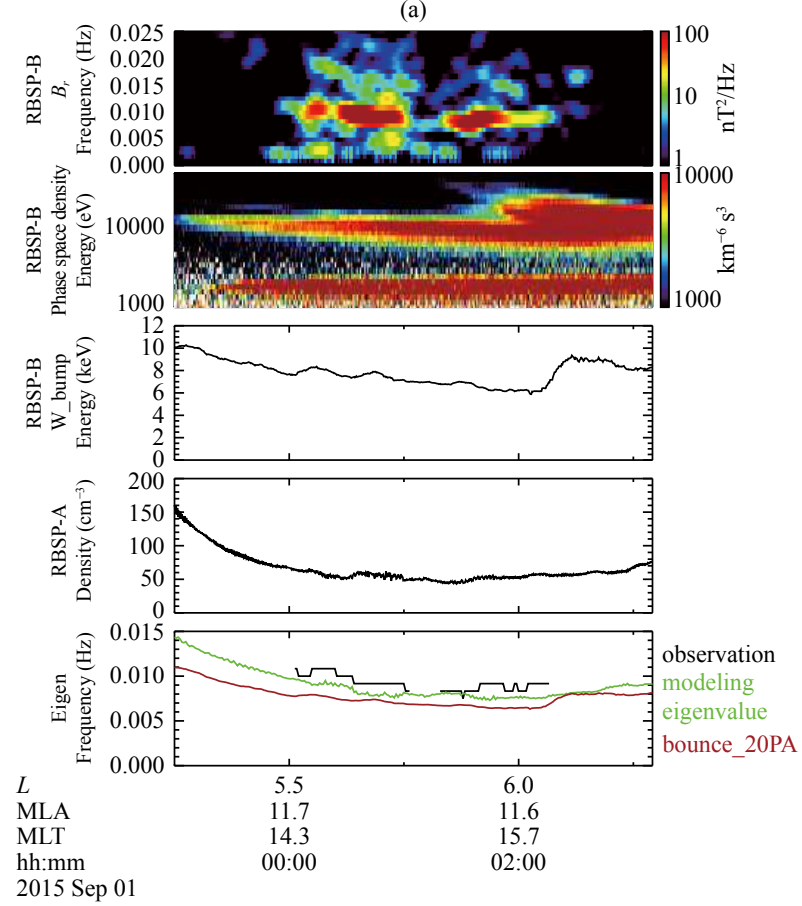

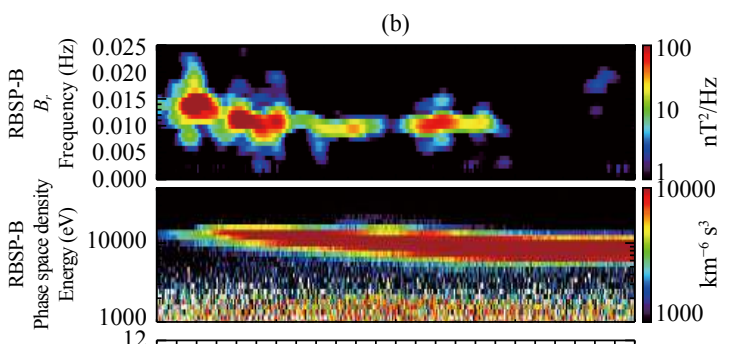

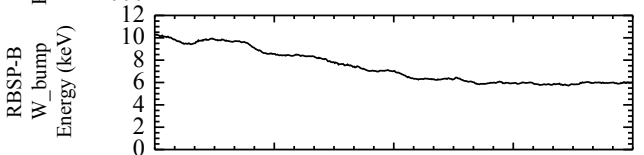

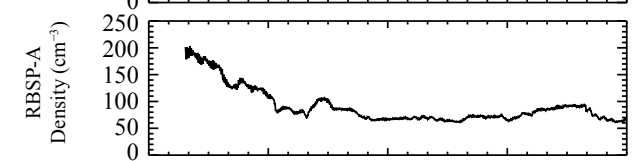

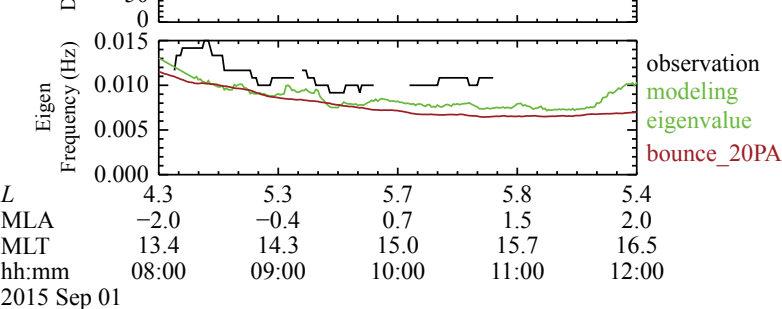

(c)

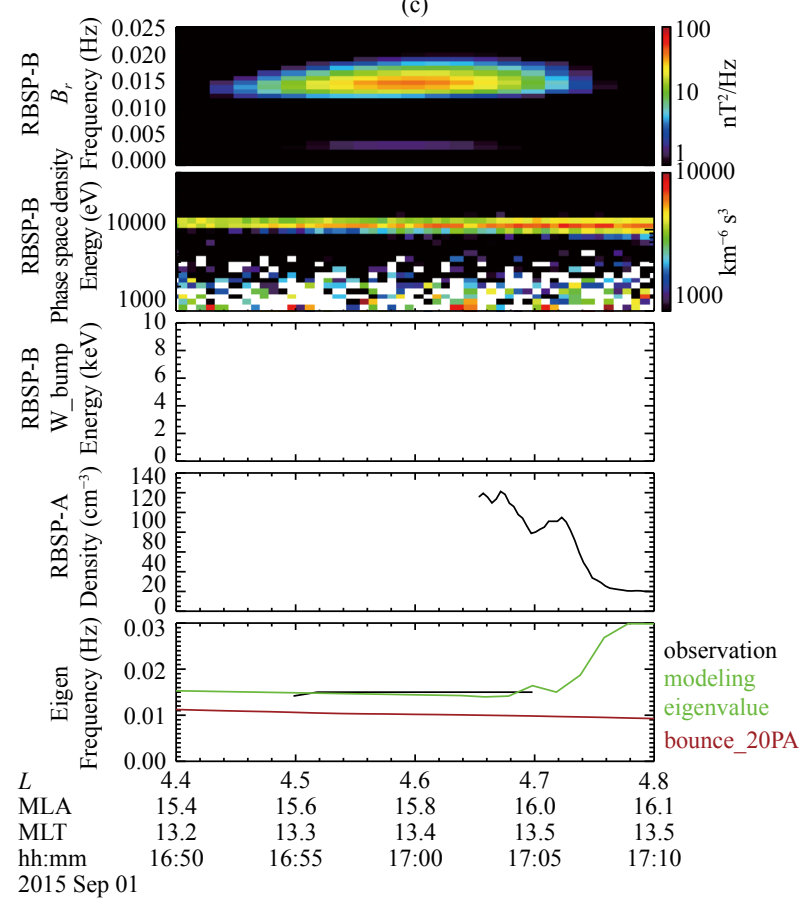

Figure 8. Observations of RBSP-B in three orbits. Panels from the top are wave power spectra of $B_{r}$, proton phase space density, energy of the bump distribution, electron density inferred from RBSP spacecraft potential, and eigenmode frequencies from the observed wave (black), from modeling of the second harmonic poloidal wave (green), and from the bounce frequency (red) of protons at the energy of the bump.

The free energy of the ULF waves may be supplied not only from bump-on-tail plasma distributions, but also from an inward gradient. According to the previous description, the generation of the poloidal wave is related to $\sim 10 \mathrm{keV}$ ions. The $\sim 10 \mathrm{keV}$ ions work by either bump-on-tail plasma distributions or by inward gradient. We will next study which mechanism provides free energy for the waves. During the particle diffusion along unique paths in W-L space from high density regions to low density regions, wave in- stability is given by

$$
\frac{\mathrm{d} f}{\mathrm{~d} W}=\left.\frac{\partial f}{\partial W}\right|_{\mu, L}+\left.\frac{\mathrm{d} L}{\mathrm{~d} W} \frac{\partial f}{\partial L}\right|_{\mu, W}>0,
$$

which condition is equivalent to

$$
\left.\frac{\mathrm{d} f}{\mathrm{~d} L}\right|_{\mu, J}=\left.\frac{\partial f}{\partial L}\right|_{\mu, W}+\left.\frac{\mathrm{d} W}{\mathrm{~d} L} \frac{\partial f}{\partial W}\right|_{\mu, L}<0
$$


(Southwood et al., 1969) by multiplying both sides by $\frac{\mathrm{d} W}{\mathrm{~d} L}$, where $f$ is the particle distribution function, $\mu$ is the first invariant, and $J$ is the second invariant. $\left.\frac{\partial f}{\partial W}\right|_{\mu, L}>0$ represents the bump-on-tail distributions while $\left.\frac{\mathrm{d} f}{\mathrm{~d} L}\right|_{\mu, W}<0$ represents inward gradients distributions. And

$$
\frac{\mathrm{d} L}{\mathrm{~d} W} \approx \frac{L^{2}}{e B_{\mathrm{E}} \omega_{d} R_{\mathrm{E}}^{2}}
$$

where $e$ is the elementary charge, $B_{\mathrm{E}}$ is the equatorial magnetic

(a)

RBSP-B_2015-08-31_23:00 UT_to_2015-09-01_01:10 UT

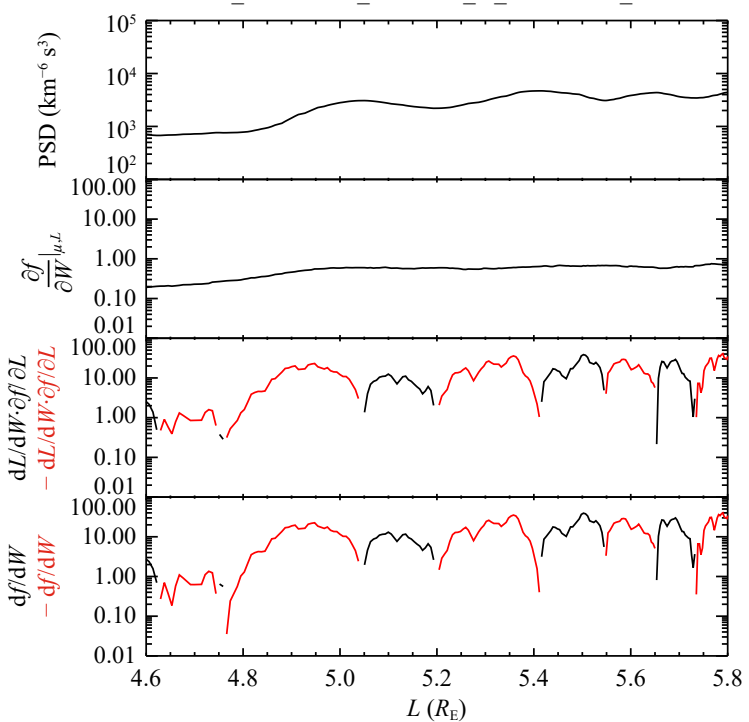

(c)

RBSP-B_2015-09-01_08:00 UT_to_2015-09-01_10:30 UT

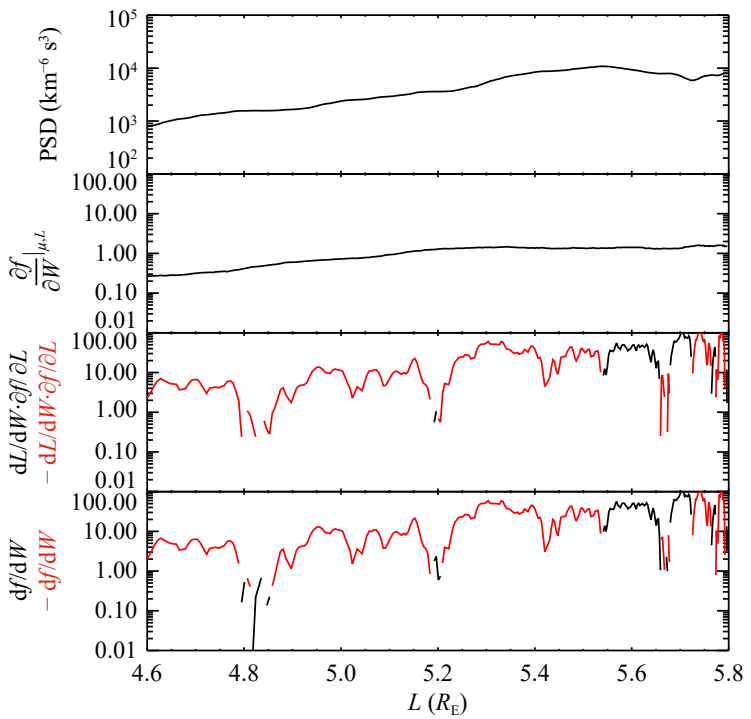

field magnitude at the surface of the Earth, and

$$
\omega_{d}=\frac{3 L W(0.35+0.15 \sin \alpha)}{\pi B_{\mathrm{E}} R_{\mathrm{E}}^{2}} .
$$

Figure 9 shows the profile of phase space density (PSD), $\left.\frac{\partial f}{\partial W}\right|_{\mu, L}$, $\left.\frac{\mathrm{d} L}{\mathrm{~d} W} \cdot \frac{\partial f}{\partial L}\right|_{\mu, W}, \frac{\mathrm{d} f}{\mathrm{~d} W}$ fixed at $\sim 10 \mathrm{keV}$ ions versus L-shell (L). PA-dependent flux are adopted to calculate PSD here. In general, PSD, $f$, is defined in the six dimensional phase space, $(p, q)$, where $p$ and $q$ denotes position and momentum respectively. For trapped

(b)
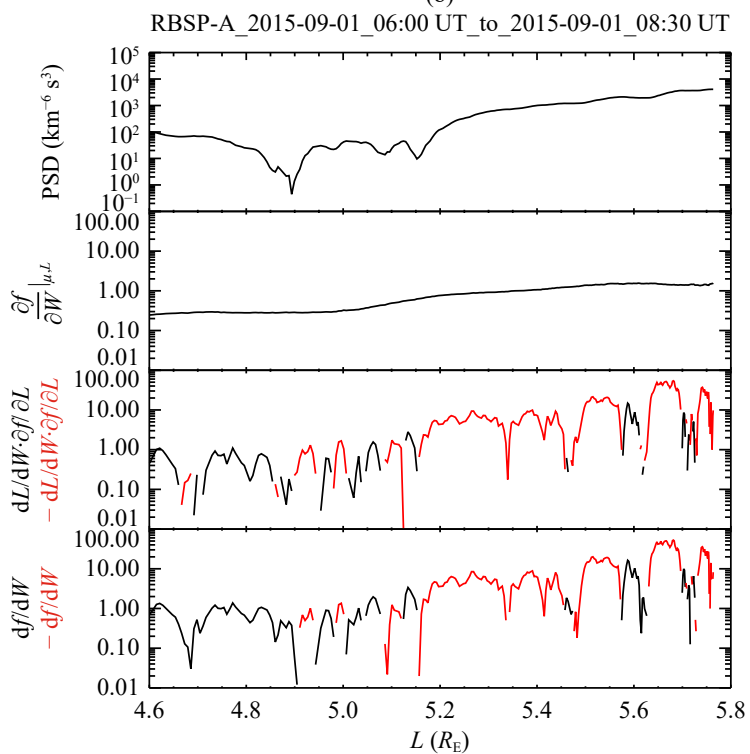

(d)

RBSP-A_2015-09-01_14:50 UT_to_2015-09-01_16:00 UT

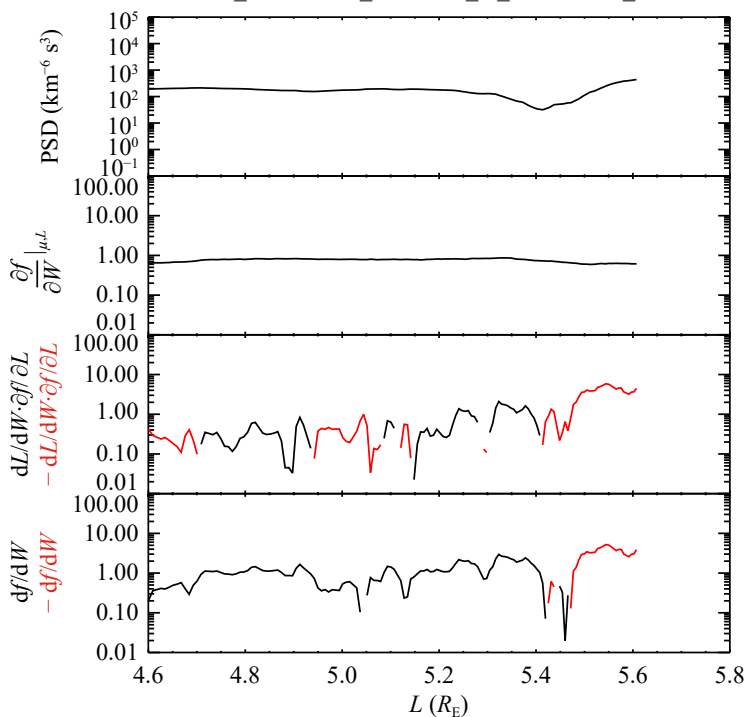

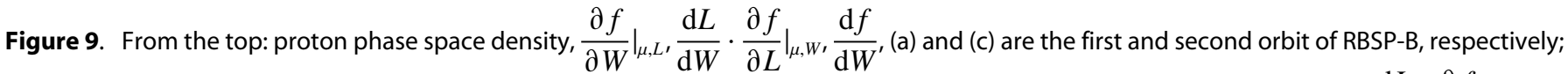
(b) and (d) are the first and second orbit of RBSP-A, respectively. In the third panel of the four pictures, the black lines represent $\left.\frac{\mathrm{d} L}{\mathrm{~d} W} \cdot \frac{\partial f}{\partial L}\right|_{\mu, W}$ and the red lines represent $-\left.\frac{\mathrm{d} L}{\mathrm{~d} W} \cdot \frac{\partial f}{\partial L}\right|_{\mu, W}$. In the fourth panel of the four pictures, the black lines represent $\frac{\mathrm{d} f}{\mathrm{~d} W}$, and the red lines represent $-\frac{\mathrm{d} f}{\mathrm{~d} W}$ 
particles, $f(p, q)$ can be alternatively written as $f\left(\mu, J, L, \phi_{1}, \phi_{2}, \phi_{3}\right.$ are action angles corresponding to the periodic motions (Green and Kivelson, 2004). However, our event occurred during storm quiet time and the quiet time was maintained for more than 48 hours before the observation of waves. Hence particles have drifted for tens of hours, much longer than the drift period of keV particless. The system may reach a steady distribution in the $\phi_{3}$ direction, and PSD may be insensitive to $\phi$. So we think the dependence on MLT can be neglected. The range of $L$ is from 4.6 to 5.8. Figures $9 a$ and $c$ represent the first and the second observation orbits of RBSP-B while Figures $9 b$ and $9 d$ represent the first and second observation orbits of RBSP-A. To examine whether the waves can gain energy from the protons in drift-bounce resonance, we should check the sign of $\frac{\mathrm{d} f}{\mathrm{~d} W}$.

Apparently, $\left.\frac{\partial f}{\partial W}\right|_{\mu, L}$ was larger than 0 at all times studied, as shown in the second panel of Figures 9a-d. As shown in the first panel of Figures 9a-d, the values of $\left.\frac{\partial f}{\partial L}\right|_{\mu, W}$ are more often positive than negative. Considering that $\frac{\mathrm{d} L}{\mathrm{~d} W}$ is negative, the values of $\left.\frac{\mathrm{d} L}{\mathrm{~d} W} \cdot \frac{\partial f}{\partial L}\right|_{\mu, W}$ are more often negative (red) and less often positive (black) in the third panel of Figures 9a-d. Thus, the positive $\left.\frac{\partial f}{\partial L}\right|_{\mu, W}$ counterbalances the free energy provided by $\left.\frac{\partial f}{\partial W}\right|_{\mu, L}>0$. In the first three pictures, the absolute values of $\left.\frac{\partial f}{\partial W}\right|_{\mu, L}$ are $\sim 0.1-1$ while the absolute values of $\left.\frac{\mathrm{d} L}{\mathrm{~d} W} \cdot \frac{\partial f}{\partial L}\right|_{\mu, W}$ are $\sim 0.1-100$. Accordingly, the inward radial gradient is dominant during the first three orbits. In the last picture, the absolute values of $\left.\frac{\partial f}{\partial W}\right|_{\mu, L}$ are near 0.6 while the absolute values of $\frac{\mathrm{d} L}{\mathrm{~d} W} \cdot \frac{\partial f}{\partial W}$ are between 0.01 and 10. Accordingly, bump-on-tail ions distributions dominate before $L \sim 5.2$ and the inward radial gradient dominates after $L \sim 5.2$. According to the analysis, bump-on-tail ion spectra contribute to the total growth rate $\left.\frac{\mathrm{d} f}{\mathrm{~d} L}\right|_{\mu, J}$. Bump-on-tail ion spectra are directly related to $\left.\frac{\partial f}{\partial W}\right|_{\mu, L}$ and indirectly contribute to $\left.\frac{\partial f}{\partial L}\right|_{\mu, W}$ as well. As calculated in Figure 9, bump-on-tail ion distributions produce effects on $\frac{\mathrm{d} f}{\mathrm{~d} L}$ through $\left.\frac{\partial f}{\partial W}\right|_{\mu, L}$ and $\left.\frac{\partial f}{\partial L}\right|_{\mu, W}$ together.

\section{Summary and Discussions}

We provide evidence from RBSP, MMS, THEMIS, and GOES that high- $m$ and highly poloidal ULF waves with time-varying polarization states from globally poloidal waves in the magnetosphere are observed. The polarization state variations occur during quiet solar wind and geomagnetic conditions. A global-scale poloidal wave is first observed by multiple spacecraft from 23:00 UT August 31 to 02:00 UT Septermber 01. In the following two consecutive RBSP orbits, the two RBSP spacecraft record the polarization state variations of the waves to highly poloidal waves. The ratio $\left|B_{a}\right| /\left|B_{r}\right|$ starts from 0.82 , for the initial wave, and decreases to 0.13 as the waves evolve to highly poloidal.
The poloidal waves from polarization variation are much more localized in both $L$ and MLT than the initially poloidal waves. Analysis of MMS measurements shows that the poloidal waves are of second harmonic mode with a high-m number (-232). The propagation of the ULF waves is westward. Data from RBSP particle instruments provide evidence that the second harmonic mode poloidal waves are powered by bump-on-tail ( 10 keV) ion distributions and inward radial gradients through the $N=1$ bounce resonance. The inward radial gradients are dominant at three of the four orbits. The radial gradient values are sometimes positive and sometimes negative. The negative values provide free energy for wave generation; the positive values counterbalance the free energy provided by $\left.\frac{\partial f}{\partial W}\right|_{\mu, L}>0$.

In MHD theory, poloidal Alfven waves convert to toroidal waves in a finite lifetime due to non-uniform plasmas (Radoski, 1974). The variation of polarization states toward the toroidal waves have been supported by simulations (Mann and Wright, 1995) and observations (Sarris et al., 2009). To our knowledge, our study presents, for the first time, observational evidence of a variation of polarization states toward purely poloidal polarity. Free energy in the energetic ions may be important for understanding this unusual variation of polarization state. The mixed polarity has a finite azimuthal wave electric field which makes it easier to initialize drift-bounce instability. Then, the drift-bounce resonance instability further increases the poloidal component of the ULF waves and supports the variation of polarization state. Although not considered in the MHD theory of Radoski (1974), such kinetic physics may effectively promote variation of polarization states leading to poloidal polarity.

The event in this study may be not uncommon. Many previous studies indicate that kinetic free energy in energetic ions can excite poloidal ULF waves through drift resonance and drift-bounce resonance (Hughes et al., 1978; Oimatsu et al., 2018; Ren J et al., 2017). Non-Maxwellian distributions are common in energetic ions (10-100 keV) in the ring current (Baddeley et al., 2004). Driftbounce resonance can occur when the bounce frequency of the energetic ions matches the local eigen-frequency of field lines, for instance as in this event. Statistical study from RBSP shows that compressional poloidal waves are distributed evenly over all phases of storms but purely poloidal waves are distributed primarily in the late recovery phase (Dai $L$ et al., 2015). Decay of the ring current might provide free energy for the polarization state variation that leads to purely poloidal waves in the late recovery phase.

\section{Acknowledgments}

This work was supported by NNSFC grants 41574161, 41731070, and 41574159 and the Strategic Pionner Program on Space Science, Chinese Academy of Sciences, grants XDA15052500 and XDA15350201. The RBSP EMFISIS data are available at http://emfisis.physics.uiowa.edu/Flight/. The RBSP ECT data are available at http://www.rbsp-ect.lanl.gov/. MMS data are publicly available at the Science Data Center at http://lasp.colorado.edu/mms/sdc/ public/. THEMIS data are available at http://themis.ssl.berkeley. edu/data/themis/. GOES data are available at http://satdat.ngdc. 
noaa.gov/sem/goes/data/. The OMNI data are available at CDAWeb. The $A E$ and Dst data are provided by the World Data Center for Geomagnetism, Kyoto.

\section{References}

Baddeley, L. J., Yeoman, T. K., Wright, D. M., Trattner, K. J., and Kellet, B. J. (2004). A statistical study of unstable particle populations in the global ringcurrent and their relation to the generation of high $m$ ULF waves. Ann. Geophys., 22(12), 4229-4241. https://doi.org/10.5194/angeo-22-4229-2004

Berk, H. L., Breizman, B. N., and Pekker, M. (1995). Numerical simulation of bump-on-tail instability with source and sink. Phys. Plasmas, 2(8), 3007-3016. https://doi.org/10.1063/1.871198

Chen, L., and Hasegawa, A. (1974). A theory of long-period magnetic pulsations: 1. Steady state excitation of field line resonance. J. Geophys. Res., 79(7), 1024-1032. https://doi.org/10.1029/JA079i007p01024

Chen, L., and Hasegawa, A. (1991). Kinetic theory of geomagnetic pulsations: 1.Internal excitations by energetic particles. J. Geophys. Res. Space Phys., 96(A2), 1503-1512. https://doi.org/10.1029/90JA02346

Cheng, C. Z., and Lin, C. S. (1987). Eigenmode analysis of compressional waves in the magnetosphere. Geophys. Res. Lett., 14(8), 884-887. https://doi.org/10.1029/GL014i008p00884

Chi, P. J., and Le, G. (2015). Observations of magnetospheric high-m poloidal waves by ST- 5 satellites in low earth orbit during geomagnetically quiet times. J. Geophys. Res. Space Phys., 120(6), 4776-4783. https://doi.org/10.1002/2015JA021145

Cramm, R., Glassmeier, K. H., Othmer, C., Fornacon, K. H., Auster, H. U., Baumjohann, W., and Georgescu, E. (2000). A case study of a radially polarized Pc4 event observed by the Equator-S satellite. Ann. Geophys., 18(4), 411-415. https://doi.org/10.1007/s00585-000-0411-5

Cummings, W. D., O'Sullivan, R. J., and Coleman, P. J. Jr. (1969). Standing Alfvén waves in the magnetosphere. J. Geophys. Res., 74(3), 778-793. https://doi.org/10.1029/JA074i003p00778

Dai, L., Takahashi, K., Wygant, J. R., Chen, L., Bonnell, J., Cattell, C. A., Thaller, S., Kletzing, C., Smith, C. W., ... Spence, H. E. (2013). Excitation of poloidal standing Alfvén waves through drift resonance wave-particle interaction. Geophys. Res. Lett., 40(16), 4127-4132. https://doi.org/10.1002/grl.50800

Dai, L., Takahashi, K., Lysak, R., Wang, C., Wygant, J. R., Kletzing, C., Bonnell, J., Cattell, C. A., Smith, C. W., ... Chen, L. J. (2015). Storm time occurrence and spatial distribution of Pc4 poloidal ULF waves in the inner magnetosphere: A Van Allen Probes statistical study. J. Geophys. Res. Space Phys., 120(6), 4748-4762. https://doi.org/10.1002/2015JA021134

Denton, R. E., and Gallagher, D. L. (2000). Determining the mass density along magnetic field lines from toroidal eigenfrequencies. J. Geophys. Res. Space Phys., 105(A12), 27717-27725. https://doi.org/10.1029/1999JA000397

Engebretson, M. J., Zanetti, L. J., Potemra, T. A., and Acuna, M. H. (1986). Harmonically structured ULF pulsations observed by the AMPTE CCE magnetic field experiment. Geophys. Res. Lett., 13(9), 905-908. https://doi.org/10.1029/GL013i009p00905

Engebretson, M. J., Murr, D. L., Erickson, K. N., Strangeway, R. J., Klumpar, D. M., Fuselier, S. A., Zanetti, L. J., and Potemra, T. A. (1992). The spatial extent of radial magnetic pulsation events observed in the dayside near synchronous orbit. J. Geophys. Res. Space Phys., 97(A9), 13741-13758. https://doi.org/10.1029/92JA00992

Fitzenreiter, R. J., Klimas, A. J., and Scudder, J. D. (1984). Detection of bump-ontail reduced electron velocity distributions at the electron foreshock boundary. Geophys. Res. Lett., 11(5), 496-499. https://doi.org/10.1029/GL011i005p00496

Funsten, H. O., Skoug, R. M., Guthrie, A. A., MacDonald, E. A., Baldonado, J. R., Harper, R. W., Henderson, K. C., Kihara, K. H., Lake, J. E., ... Chen, J. (2013). Helium, oxygen, proton, and electron (HOPE) mass spectrometer for the radiation belt storm probes mission. Space Sci. Rev., 179(1-4), 423-484. https://doi.org/10.1007/s11214-013-9968-7

Green, J. C., and Kivelson, M. G. (2004). Relativistic electrons in the outer radiation belt: Differentiating between acceleration mechanisms. J.
Geophys. Res. Space Phys., 109(A3), A03213.

https://doi.org/10.1029/2003JA010153

Hughes, W. J., Southwood, D. J., Mauk, B., McPherron, R. L., and Barfield, J. N. (1978). Alfvén waves generated by an inverted plasma energy distribution. Nature, 275(5675), 43-45. https://doi.org/10.1038/275043a0

Hughes, W. J. (1994). Magnetospheric ULF waves: a tutorial with a historical perspective. In M. J., Engebretson, et al. (Eds.), Solar Wind Sources of Magnetospheric Ultra-Low-Frequency Waves. Washington: American Geophysical Union. https://doi.org/10.1029/GM081p0001

Kessel, R. L. (2008). Solar wind excitation of Pc5 fluctuations in the magnetosphere and on the ground. J. Geophys. Res. Space Phys., 113(A4), A04202. https://doi.org/10.1029/2007JA012255

Kletzing, C. A., Kurth, W. S., Acuna, M., MacDowall, R. J., Torbert, R. B., Averkamp, T., Bodet, D., Bounds, S. R., Chutter, M., ... Tyler, J. (2013). The electric and magnetic field instrument suite and integrated science (EMFISIS) on RBSP. Space Sci. Rev., 179(1-4), 127-181. https://doi.org/10.1007/s11214-0139993-6

Klimushkin, D. Y. (1998). Resonators for hydromagnetic waves in the magnetosphere. J. Geophys. Res. Space Phys., 103(A2), 2369-2375. https://doi.org/10.1029/97JA02193

Klimushkin, D. Y., Mager, P. N., and Glassmeier, K. H. (2004). Toroidal and poloidal alfvén waves with arbitrary azimuthal wavenumbers in a finite pressure plasma in the earth's magnetosphere. Ann. Geophys., 22(1), 267-287. https://doi.org/10.5194/angeo-22-267-2004

Le, G., Chi, P. J., Strangeway, R. J., and Slavin, J. A. (2011). Observations of a unique type of ULF wave by low-altitude Space Technology 5 satellites. J. Geophys. Res. Space Phys., 116(A8), A08203. https://doi.org/10.1029/2011JA016574

Le, G., Chi, P. J., Strangeway, R. J., Russell, C. T., Slavin, J. A., Takahashi, K., Singer, H. J., Anderson, B. J., Bromund, K., ... Torbert, R. B. (2017). Global observations of magnetospheric high- $m$ poloidal waves during the 22 June 2015 magnetic storm. Geophys. Res. Lett., 44(8), 3456-3464. https://doi.org/10.1002/2017GL073048

Leonovich, A. S., and Mazur, V. A. (1995). Magnetospheric resonator for transverse-small-scale standing Alfvén waves. Planet. Space Sci., 43(7), 881-883. https://doi.org/10.1016/0032-0633(94)00206-7

Li, L., Zhou, X. Z., Zong, Q. G., Rankin, R., Zou, H., Liu, Y., Chen, X. R., and Hao, Y. $X$. (2017). Charged particle behavior in localized ultralow frequency waves: Theory and observations. Geophys. Res. Lett., 44(12), 5900-5908. https://doi.org/10.1002/2017GL073392

Lindqvist, P. A., Olsson, G., Torbert, R. B., King, B., Granoff, M., Rau, D., Needell, G., Turco, S., Dors, I., ... Åhlén, L. (2016). The spin-plane double probe electric field instrument for MMS. Space Sci. Rev., 199(1-4), 137-165. https://doi.org/10.1007/s11214-014-0116-9

Liu, W., Sarris, T. E., Li, X., Zong, Q. G., Ergun, R., Angelopoulos, V., and Glassmeier, K. H. (2011). Spatial structure and temporal evolution of a dayside poloidal ULF wave event. Geophys. Res. Lett., 38(19), L19104. https://doi.org/10.1029/2011GL049476

Liu, W., Cao, J. B., Li, X., Sarris, T. E., Zong, Q. G., Hartinger, M., Takahashi, K., Zhang, H., Shi, Q. Q., and Angelopoulos, V. (2013). Poloidal ULF wave observed in the plasmasphere boundary layer. J. Geophys. Res. Space Phys., 118(7), 4298-4307. https://doi.org/10.1002/jgra.50427

Mager, P. N., and Klimushkin, D. Y. (2013). Giant pulsations as modes of a transverse Alfvénic resonator on the plasmapause. Earth Planets Space, 65(5), 397-409. https://doi.org/10.5047/eps.2012.10.002

Mann, I. R., and Wright, A. N. (1995). Finite lifetimes of ideal poloidal Alfvén waves. J. Geophys. Res. Space Phys., 100(A12), 23677-23686. https://doi.org/10.1029/95JA02689

Menk, F. W., Orr, D., Clilverd, M. A., Smith, A. J., Waters, C. L., Milling, D. K., and Fraser, B. J. (1999). Monitoring spatial and temporal variations in the dayside plasmasphere using geomagnetic field line resonances. J. Geophys. Res. Space Phys., 104(A9), 19955-19. https://doi.org/10.1029/1999JA900205

Min, K., Takahashi, K., Ukhorskiy, A. Y., Manweiler, J. W., Spence, H. E., Singer, H., J., Claudepierre, S. G., Larsen, B. A., Soto-Chavez, B. A., ... Cohen, R. J. (2017). Second harmonic poloidal waves observed by Van Allen Probes in the dusk- 
midnight sector. J. Geophys. Res. Space Phys., 122(3), 3013-3039. https://doi.org/10.1002/2016JA023770

Oimatsu, S., Nosé, M., Takahashi, K., Yamamoto, K., Keika, K., Kletzing, C. A., Smith, C. W., MacDowall, R. J., and Mitchell, D. G. (2018). Van Allen probes observations of drift-bounce resonance and energy transfer between energetic ring current protons and poloidal Pc4 wave. J. Geophys. Res. Space Phys., 123(5), 3421-3435. https://doi.org/10.1029/2017JA025087

Orr, D., and Matthew, J. A. D. (1971). The variation of geomagnetic micropulsation periods with latitude and the plasmapause. Planet. Space Sci., 19(8), 897-905. https://doi.org/10.1016/0032-0633(71)90141-3

Radoski, H. R. (1974). A theory of latitude dependent geomagnetic micropulsations: The asymptotic fields. J. Geophys. Res., 79(4), 595-603. https://doi.org/10.1029/JA079i004p00595

Ren, J., Zong, Q. G., Miyoshi, Y., Zhou, X. Z., Wang, Y. F., Rankin, R., Yue, C., Spence, H. E., Funsten, H. O., ... Kletzing, C. A. (2017). Low-energy (<200 eV) electron acceleration by ULF waves in the plasmaspheric boundary layer: Van Allen probes observation. J. Geophys. Res. Space Phys., 122(10), 9969-9982. https://doi.org/10.1002/2017JA024316

Roederer, J. G., and Hones, E. W. Jr. (1970). Electric field in the magnetosphere as deduced from asymmetries in the trapped particle flux. J. Geophys. Res., 75(19), 3923-3926. https://doi.org/10.1029/JA075i019p03923

Russell, C. T., Luhmann, J. G., Odera, T. J., and Stuart, W. F. (1983). The rate of occurrence of dayside Pc 3, 4 pulsations: The L-value dependence of the IMF cone angle effect. Geophys. Res. Lett., 10(8), 663-666. https://doi.org/10.1029/GL010i008p00663

Russell, C. T., Anderson, B. J., Baumjohann, W., Bromund, K. R., Dearborn, D., Fischer, D., Le, G., Leinweber, H. K., Leneman, D., ... Richter, I. (2016). The magnetospheric multiscale magnetometers. Space Sci. Rev., 199(1-4), 189-256. https://doi.org/10.1007/s11214-014-0057-3

Sarris, T. E., Wright, A. N., and Li, X. (2009). Observations and analysis of Alfvén wave phase mixing in the Earth's magnetosphere. J. Geophys. Res. Space Phys., 114(A3), A03218. https://doi.org/10.1029/2008JA013606

Schäfer, S., Glassmeier, K. H., Eriksson, P. T. I., Pierrard, V., Fornaçon, K. H., and Blomberg, L. G. (2007). Spatial and temporal characteristics of poloidal waves in the terrestrial plasmasphere: a CLUSTER case study. Ann. Geophys., 25(4), 1011-1024. https://doi.org/10.5194/angeo-25-1011-2007

Singer, H. J., Hughes, W. J., and Russell, C. T. (1982). Standing hydromagnetic waves observed by ISEE 1 and 2: Radial extent and harmonic. J. Geophys. Res. Space Phys., 87(A5), 3519-3529. https://doi.org/10.1029/JA087iA05p03519

Southwood, D. J., Dungey, J. W., and Etherington, R. J. (1969). Bounce resonant interaction between pulsations and trapped particles. Planet. Space Sci., 17(3), 349-361. https://doi.org/10.1016/0032-0633(69)90068-3

Southwood, D. J. (1974). Some features of field line resonances in the magnetosphere. Planet. Space Sci., 22(3), 483-491. https://doi.org/10.1016/0032-0633(74)90078-6

Southwood, D. J. (1976). A general approach to low-frequency instability in the ring current plasma. J. Geophys. Res., 81(19), 3340-3348. https://doi.org/10.1029/JA081i019p03340

Southwood, D. J., and Kivelson, M. G. (1982). Charged particle behavior in lowfrequency geomagnetic pulsations, 2. Graphical approach. J. Geophys. Res. Space Phys., 87(A3), 1707-1710. https://doi.org/10.1029/JA087iA03p01707 Takahashi, K., McEntire, R. W., Lui, A. T. Y., and Potemra, T. A. (1990). Ion flux oscillations associated with a radially polarized transverse Pc 5 magnetic pulsation. J. Geophys. Res. Space Phys., 95(A4), 3717-3731.

https://doi.org/10.1029/JA095iA04p03717

Takahashi, K., Glassmeier, K. H., Angelopoulos, V., Bonnell, J., Nishimura, Y., Singer, H. J., and Russell, C. T. (2011). Multisatellite observations of a giant pulsation event. J. Geophys. Res. Space Phys., 116(A11), A11223. https://doi.org/10.1029/2011JA016955

Takahashi, K., Denton, R. E., Kurth, W., Kletzing, C., Wygant, J., Bonnell, J., Dai, L., Min, K., Smith, C. W., and MacDowall, R. (2015). Externally driven plasmaspheric ulf waves observed by the Van Allen probes. J. Geophys. Res. Space Phys., 120(1), 526-552. https://doi.org/10.1002/2014JA020373

Takahashi, K., Claudepierre, S. G., Rankin, R., Mann, I. R., and Smith, C. W. (2018a). Van Allen probes observation of a fundamental poloidal standing Alfvén wave event related to giant pulsations. J. Geophys. Res. Space Phys., 123(6), 4574-4593. https://doi.org/10.1029/2017JA025139

Takahashi, K., Oimatsu, S., Nosé, M., Min, K., Claudepierre, S. G., Chan, A., Wygant, J., and Kim, H. (2018b). Van Allen probes observations of second harmonic poloidal standing Alfvén waves. J. Geophys. Res. Space Phys., 123(1), 611-637. https://doi.org/10.1002/2017JA024869

Torbert, R. B., Russell, C. T., Magnes, W., Ergun, R. E., Lindqvist, P. A., LeContel, O., Vaith, H., Macri, J., Myers, ... Lappalainen, K. (2016). The FIELDS instrument suite on MMS: Scientific objectives, measurements, and data products. Space Sci. Rev., 199(1-4), 105-135. https://doi.org/10.1007/s11214-0140109-8

Wygant, J. R., Bonnell, J. W., Goetz, K., Ergun, R. E., Mozer, F. S., Bale, S. D., Ludlam, M., Turin, P., Harvey, P. R., ... Tao, J. B. (2013). The electric field and waves instruments on the radiation belt storm probes mission. Space Sci. Rev., 179(1-4), 183-220. https://doi.org/10.1007/s11214-013-0013-7

Yeoman, T. K., Wright, D. M., Chapman, P. J., and Stockton-Chalk, A. B. (2000). High-latitude observations of ULF waves with large azimuthal wavenumbers. J. Geophys. Res. Space Phys., 105(A3), 5453-5462. https://doi.org/10.1029/1999JA005081

Zhao, L. L., Zhang, H., and Zong, Q. G. (2017). Global ULF waves generated by a hot flow anomaly. Geophys. Res. Lett., 44(11), 5283-5291. https://doi.org/10.1002/2017GL073249

Zhou, X. Z., Wang, Z. H., Zong, Q. G., Claudepierre, S. G., Mann, I. R., Kivelson, M. G., Angelopoulos, V., Hao, Y. X., Wang, Y. F., and Pu, Z. Y. (2015). Imprints of impulse-excited hydromagnetic waves on electrons in the Van Allen radiation belts. Geophys. Res. Lett., 42(15), 6199-6204. https://doi.org/10.1002/2015GL064988

Zong, Q. G., Zhou, X. Z., Li, X., Song, P., Fu, S. Y., Baker, D. N., Pu, Z. Y., Fritz, T. A., Daly, ... Réme, H. (2007). Ultralow frequency modulation of energetic particles in the dayside magnetosphere. Geophys. Res. Lett., 34(12), L12105. https://doi.org/10.1029/2007GL029915

Zong, Q. G., Zhou, X. Z., Wang, Y. F., Li, X., Song, P., Baker, D. N., Fritz, T. A., Daly, P. W., Dunlop, M., and Pedersen, A. (2009). Energetic electron response to ULF waves induced by interplanetary shocks in the outer radiation belt. J. Geophys. Res. Space Phys., 114(A10), A10204. https://doi.org/10.1029/2009JA014393

Zong, Q. G., Rankin, R., and Zhou, X. Z. (2017). The interaction of ultra-lowfrequency pc3-5 waves with charged particles in earth's magnetosphere. Rev. Mod. Plasma Phys., 1(1), 10. https://doi.org/10.1007/s41614-017-0011-4 